QUARTERLY OF APPLIED MATHEMATICS

VOLUME LXVII, NUMBER 1

MARCH 2009, PAGES 47-71

S 0033-569X(09)01094-3

Article electronically published on January 8, 2009

\title{
THERMO-VISCO-ELASTICITY AT SMALL STRAINS WITH $L^{1}$-DATA
}

\author{
BY \\ TOMÁS̆ ROUBÍČEK \\ Mathematical Institute, Charles University, Sokolovská 83, CZ-186 75 Praha 8, Czech Republic \\ Institute of Thermomechanics of the ASCR, Dolejškova 5, CZ-182 00 Praha 8, Czech Republic
}

\begin{abstract}
Existence of a very weak solution to the $d$-dimensional thermo-viscoelasticity system for Kelvin-Voigt-type material at small strains involving (possibly nonlinear) monotone viscosity of a $p$-Laplacian type and temperature-dependent heat capacity of an $(\omega-1)$-polynomial growth is proved by a successive passage to a limit in a suitably regularized Galerkin approximation and sophisticated a priori estimates for the temperature gradient performed for the coupled system. A global solution for arbitrarily large data having an $L^{1}$-structure is obtained under the conditions $p \geq 2, \omega \geq 1$, and $p>1+d /(2 \omega)$.
\end{abstract}

1. Introduction. Existence of a solution of a coupled thermo-visco-elasticity system for Kelvin-Voigt-type material with a linear dissipation and the heat equation with constant heat capacity and conductivity is, in spite of great effort through many decades, still open in dimensions $d \geq 2$, while in dimension $d=1$ it was proved already in [8, 9] in 1982. Investigation of one-dimensional problems continued, e.g., in [5, 6, 7, 13, 14, 16, 20, 24, 26, 29, 30, 31, 32, 33.

The literature about multidimensional problems is more modest, however. The linear dissipation mechanism in the case $d \geq 2$ would need suitable modifications in the heat equation. One option involving a nonconstant heat capacity depending on the temperature $\theta$ with a growth at least $\theta^{1 / 2+\varepsilon}, \varepsilon>0$, was considered in [2], where the existence of a solution has been proved by a Schauder fixed point. A similar Schauder fixed method was also used in 34, 35.

Alternatively, keeping the heat capacity constant, one can modify the heat conductivity to make it dependent either on $\theta$ (as in [10] with a growth greater than $1-2 / d$, see also

Received June 4, 2007.

2000 Mathematics Subject Classification. Primary 74F05; Secondary 35K55, 74H20, 80A17.

Key words and phrases. Thermoviscoelasticity, Kelvin-Voigt materials, very weak solutions.

This work was created as a research activity of "Nečas center for mathematical modeling" LC 06052 (MŠMT ČR) partly supported also by the grants IAA 1075402 (GA AV ČR) and MSM 21620839 and 1M06031 (MŠMT ČR) and the research plan AVOZ20760514 (ČR). The author acknowledges comments of Dr. Giusseppe Tomassetti that improved the previous version at a lot of places.

E-mail address: tomas.roubicek@mff.cuni.cz 
[11, Sect.5.4.2.1]) or on $\nabla \theta$ (as in [11, Sect.5.4.2.2] or [15] with a growth $\geq d / 2-1$ if $d \leq 3$ ). For the last case, see also [18, 19] where a growth condition $2 s \kappa^{\prime}(s)+\kappa(s) \geq \varepsilon(1+s)$ imposed on the heat conductivity $\kappa=\kappa\left(|\nabla \theta|^{2}\right)$ was assumed. In the latter works, regular data had been required. Another modification of the heat flux, namely by a term $\frac{\partial}{\partial t} \nabla \theta$, was used in [20, again for the one-dimensional case only.

Another possibility for handling linear dissipation is to involve higher-order terms of the type $\left|\nabla^{2} u\right|^{2}$ into the stored energy which then can even be nonconvex in terms of strain; cf. [13, 21, 23, 26, 30, 31] or also [5, Chap.5].

Alternatively, the linear dissipation mechanism can be strengthened by some higherorder "capillarity-like" terms such as $\Delta^{2} \frac{\partial}{\partial t} u$ or some spatially nonlocal term; cf. Remark 4.10 below.

The goal is to prove the existence of a solution to the thermoviscoelastic system by a constructive method without using Schauder-fixed-point-type arguments and under extreme loading having only the basic integrability of heating and mechanical forcing. Also, a combination of nonlinearities in the heat equation (as in [2]) and in the dissipation reveals a proper condition (3.2) on their growth.

2. Thermo-visco-elastic system. We assume a body occupying the bounded domain $\Omega$ with a Lipschitz boundary $\Gamma$, made from Kelvin-Voigt-type viscoelastic and heat-conductive material described in terms of the small strains. Considering a fixed time horizon $T>0$, we abbreviate $Q:=(0, T) \times \Omega$ and $\Sigma:=(0, T) \times \Gamma$. The governing equations for the displacement $u$ and temperature $\theta$ consist of the equilibrium equation that balances the total stress $\sigma$ with the inertial forces and the outer loading by a body force $f$ :

$$
\begin{aligned}
\rho \frac{\partial^{2} u}{\partial t^{2}}-\operatorname{div} \sigma & =f \quad \text { with } \\
\sigma & =\mathbb{C}(e(u)-\theta \mathbb{B})+\mathbb{D}\left(\frac{\partial e(u)}{\partial t}\right), \quad e(u)=\frac{1}{2} \nabla u^{\top}+\frac{1}{2} \nabla u,
\end{aligned}
$$

and the heat equation that balances the total energy, cf. (4.57) below,

$$
\mathfrak{c}(\theta) \frac{\partial \theta}{\partial t}-\operatorname{div}(\mathbb{K} \nabla \theta)=\mathbb{D}\left(\frac{\partial e(u)}{\partial t}\right): \frac{\partial e(u)}{\partial t}-\theta \mathbb{C B}: \frac{\partial e(u)}{\partial t}+g,
$$

where the data in this model are:

$u: Q \rightarrow \mathbb{R}^{d}$ displacement,

$\theta: Q \rightarrow \mathbb{R}$ temperature,

$e(u): Q \rightarrow \mathbb{R}_{\mathrm{sym}}^{d \times d}$ the small-strain tensor,

$\mathbb{C}$ the 4 th-order tensor of elastic constants,

$\mathbb{D}: \mathbb{R}_{\text {sym }}^{d \times d} \rightarrow \mathbb{R}_{\text {sym }}^{d \times d}$ a (possibly nonlinear) viscosity,

$\mathbb{B} \in \mathbb{R}_{\text {sym }}^{d \times d}$ the thermal-dilation tensor,

$\mathfrak{c}>0$ heat capacity (depending on $\theta$ ),

$\rho>0$ mass density,

$\mathbb{K} \in \mathbb{R}_{\text {sym }}^{d \times d}$ the heat-conductivity tensor,

$f: Q \rightarrow \mathbb{R}^{d}$ the body force,

$g: Q \rightarrow \mathbb{R}$ the prescribed body heat source, and

$h: \Sigma \rightarrow \mathbb{R}$ (in (2.3a) below) the prescribed boundary flux, 
where $\mathbb{R}_{\text {sym }}^{d \times d}$ is the set of symmetric $d \times d$ matrices. By $3.3 \mathrm{~b}$ below, $\mathbb{C}$ induces a linear mapping $\mathbb{R}_{\mathrm{sym}}^{d \times d} \rightarrow \mathbb{R}_{\mathrm{sym}}^{d \times d}$ defined by $e \mapsto\left[\sum_{k, l=1}^{d} \mathbb{C}_{i j k l} e_{k l}\right]$.

We will consider an initial-boundary-value problem for the system (2.1)-(2.2) and therefore we choose some boundary conditions, e.g. an unsupported body heated by an external flux $h$, and initial conditions:

$$
\begin{aligned}
& \begin{array}{lll}
\nu \cdot \sigma=0, & \mathbb{K} \frac{\partial \theta}{\partial \nu}=h \quad \text { on } \Sigma,
\end{array} \\
& u(0, \cdot)=u_{0}, \quad \frac{\partial u}{\partial t}(0, \cdot)=v_{0}, \quad \theta(0, \cdot)=\theta_{0} \quad \text { on } \Omega .
\end{aligned}
$$

REMARK 2.1 (Thermodynamics of the model). The model (2.1)-(2.2) can be derived from the specific Helmholtz free energy considered as

$$
\psi(\theta, e):=\frac{1}{2} \mathbb{C}(e-\mathbb{B} \theta):(e-\mathbb{B} \theta)-\frac{\theta^{2}}{2} \mathbb{C B}: \mathbb{B}-\psi_{0}(\theta) .
$$

The particular terms in (2.4) are related respectively to the elastic stored energy, temperature dilation, and a contribution of chaotic vibrations of the atomic grid. Moreover, we pose the dissipation rate:

$$
\xi(\dot{e}):=\mathbb{D}(\dot{e}): \dot{e}, \quad \dot{e}=\frac{\partial e(u)}{\partial t} .
$$

The form of $\xi$ is related to (possibly nonlinear) viscosity. Defining still the specific entropy by the so-called Gibbs' relation $s=s(\theta, e)=-\psi_{\theta}^{\prime}(\theta, e)=\mathbb{C B}: e+\psi_{0}^{\prime}(\theta)$ and the heat flux $j:=-\mathbb{K} \nabla \theta$ by Fourier's law (in an anisotropic medium), the so-called entropy equation

$$
\theta \frac{\partial s}{\partial t}=\xi\left(\frac{\partial e(u)}{\partial t}\right)-\operatorname{div}(j)+g
$$

yields, after some calculus, the heat equation (2.2) with the heat capacity

$$
\mathfrak{c}(\theta)=\theta \psi_{0}^{\prime \prime}(\theta) .
$$

Furthermore, the total stress is postulated in the Kelvin-Voigt rheology as

$$
\sigma=\psi_{e}^{\prime}(\theta, e(u))+\mathbb{D}\left(\frac{\partial e(u)}{\partial t}\right)
$$

which just gives (2.10). From Hamilton's principle using the specific kinetic energy $\frac{1}{2} \rho\left|\frac{\partial}{\partial t} u\right|^{2}$ generalized for dissipative systems, one then obtains the equilibrium equation (2.17). The entropy equation (2.6) is designed to balance the total energy, i.e. the sum of the internal energy

$$
\psi+\theta s=\theta \psi_{0}^{\prime}(\theta)-\psi_{0}(\theta)+\frac{1}{2} \mathbb{C} e(u): e(u)-\theta \mathbb{C B}: e(u)
$$

and the kinetic energy integrated over $\Omega$ with the overall dissipated energy; cf. (4.57) below. Assuming $g \geq 0, h \geq 0$, and $u_{0}>0$, we can at least formally rely on $\theta>0$ and, using (2.6) and (3.3k) below, derive the Clausius-Duhem inequality

$$
\begin{aligned}
\frac{\mathrm{d}}{\mathrm{d} t} \int_{\Omega} s \mathrm{~d} x & =\int_{\Omega} \operatorname{div}\left(\mathbb{K} \frac{\nabla \theta}{\theta}\right)+\frac{\mathbb{K} \nabla \theta \cdot \nabla \theta}{\theta^{2}}+\frac{g}{\theta} \mathrm{d} x \\
& =\int_{\Omega} \frac{\mathbb{K} \nabla \theta \cdot \nabla \theta}{\theta^{2}}+\frac{g}{\theta} \mathrm{d} x+\int_{\Gamma} \frac{h}{\theta} \mathrm{d} S \geq 0 .
\end{aligned}
$$


A special case $\psi_{0}(\theta)=c_{0} \theta \ln \left(\theta / \theta_{0}\right)$ with $c_{0}>0$ and $\theta_{0}$ constant gives $\mathfrak{c}(\theta)=c_{0}$ in (2.7) and the thermal part of the internal energy (2.9) as $\theta \psi_{0}^{\prime}(\theta)-\psi_{0}(\theta)=c_{0} \theta$.

EXAMPLE 2.2 (Isotropic material). The above general setting allows for general anisotropic materials as single crystals or composites. In special cases such as cubic or tetragonal single crystals or stratified composites, more symmetries can be required in $\mathbb{B}$, $\mathbb{C}, \mathbb{D}$, and $\mathbb{K}$ than those assumed in (3.3) below. The extreme case is isotropic where $\mathbb{B}=\operatorname{diag}(\alpha, \ldots, \alpha)$ and $\mathbb{K}=\operatorname{diag}(\kappa, \ldots, \kappa)$ with $\alpha$ thermal dilatability and $\kappa>0$ heat conductivity, and only two coefficients, denoted as $\lambda \geq 0$ and $\mu>0$ (called Lamé constants) occur in $\mathbb{C}$, and similarly for $\mathbb{D}$ where some nonlinear dependence is to be considered if $p \neq 2$; the specific form (2.11) is just a particular example for it, of course. Thus, in this isotropic case, we have

$$
\begin{aligned}
& \mathbb{B}_{i j}=\alpha \delta_{i j}, \\
& \mathbb{C}_{i j k l}=\lambda \delta_{i j} \delta_{k l}+\mu\left(\delta_{i k} \delta_{j l}+\delta_{i l} \delta_{j k}\right), \\
& \mathbb{D}(\dot{e})=\mathbb{D}^{0}(\dot{e}) \dot{e} \quad \text { with } \quad \mathbb{D}_{i j k l}^{0}(\dot{e})=\left(\lambda^{0}+\lambda^{1}\left(|\operatorname{tr}(\dot{e})|^{p-2}\right) \delta_{i j} \delta_{k l}\right. \\
& +\left(\mu^{0}+\mu^{1}|\dot{e}|^{p-2}\right)\left(\delta_{i k} \delta_{j l}+\delta_{i l} \delta_{j k}\right), \\
& \mathbb{K}_{i j}=\kappa \delta_{i j},
\end{aligned}
$$

where $\delta$ denotes here the Kronecker symbol, "tr" denotes the trace of a matrix and $\lambda^{0}, \lambda^{1}, \mu^{0} \geq 0$ and $\mu^{1} \geq 0$. Note that the stored energy and the dissipation rate take respectively the form

$$
\begin{aligned}
& \frac{1}{2} \mathbb{C} e: e=\frac{\lambda}{2} \operatorname{tr}(e)^{2}+\mu|e|^{2} \quad \text { and } \\
& \mathbb{D}(\dot{e}): \dot{e}=\lambda^{0} \operatorname{tr}(\dot{e})^{2}+\lambda^{1} \operatorname{tr}(\dot{e})^{p}+2 \mu^{0}|\dot{e}|^{2}+2 \mu^{1}|\dot{e}|^{p},
\end{aligned}
$$

where $|e|$ means the Frobenius norm of the matrix $e$.

3. Data qualification, very weak solution. We assume

$$
\Omega \text { a bounded Lipschitz domain in } \mathbb{R}^{d}, d \geq 1 \text {, }
$$

and, for some exponents $p$ and $\omega$ satisfying

$$
p \geq 2, \quad \omega \geq 1, \quad \text { and } \quad p>\frac{d+2 \omega}{2 \omega},
$$


or, in other words, $\omega>d /(2 p-2)$, the following basic qualification for $\mathbb{C}, \mathbb{D}, \mathfrak{c}$ and for $\mathbb{K}:$

$$
\begin{aligned}
& \exists \eta_{0}>0 \forall e \in \mathbb{R}_{\mathrm{sym}}^{d \times d}: \quad \frac{1}{2} \mathbb{C} e: e \geq \eta_{0}|e|^{2}, \\
& \mathbb{C}_{i j k l}=\mathbb{C}_{j i k l}=\mathbb{C}_{k l i j}, \\
& \mathbb{D}: \mathbb{R}_{\mathrm{sym}}^{d \times d} \rightarrow \mathbb{R}_{\mathrm{sym}}^{d \times d} \text { continuous, } \\
& \exists \eta_{1}>0 \forall \dot{e} \in \mathbb{R}_{\mathrm{sym}}^{d \times d}: \quad \mathbb{D}(\dot{e}): \dot{e} \geq \eta_{1}|\dot{e}|^{p}, \\
& \exists C_{0} \forall \dot{e} \in \mathbb{R}_{\mathrm{sym}}^{d \times d}: \quad|\mathbb{D}(\dot{e})| \leq C_{0}\left(1+|\dot{e}|^{p-1}\right), \\
& \exists \eta_{2}>0 \forall \dot{e}_{1}, \dot{e}_{2} \in \mathbb{R}_{\mathrm{sym}}^{d \times d}: \quad\left(\mathbb{D}\left(\dot{e}_{1}\right)-\mathbb{D}\left(\dot{e}_{2}\right)\right):\left(\dot{e}_{1}-\dot{e}_{2}\right) \geq \eta_{2}\left|\dot{e}_{1}-\dot{e}_{2}\right|^{p}, \\
& \mathfrak{c}: \mathbb{R}^{+} \rightarrow \mathbb{R} \text { continuously differentiable, } \\
& \exists c_{\max } \geq c_{\min }>0, \quad \forall \theta \in \mathbb{R}^{+}: \quad c_{\min }(1+\theta)^{\omega-1} \leq \mathfrak{c}(\theta) \leq c_{\max }\left(1+\theta^{\omega-1}\right), \\
& \exists \zeta>0 \quad \exists C_{\max } \in \mathbb{R}: \quad \mathfrak{c}^{\prime}(\theta) \leq C_{\max } \frac{\mathfrak{c}(\theta)^{2}}{(1+\theta)^{1+\zeta}}, \\
& \mathbb{K} \in \mathbb{R}_{\mathrm{sym}}^{d \times d}, \\
& \exists \kappa>0 \quad \forall z \in \mathbb{R}^{d}: \quad \mathbb{K} z \cdot z \geq \kappa|z|^{2} .
\end{aligned}
$$

An example for $\mathfrak{c}$ satisfying $(3.3 \mathrm{~g}, \mathrm{~h}, \mathrm{i})$ is $\mathfrak{c}(\theta):=c_{\min }(1+\theta)^{\omega-1}$ with any $\omega \geq 1$. For such a $\mathfrak{c}$, (3.3i) holds with $\zeta \leq \omega-1$ if $\omega>1$ while for $\omega=1$ such a $\mathfrak{c}$ is constant and $\mathfrak{c}^{\prime}=0$; hence (3.3i) holds too. The rather nonconventional condition (3.3i) will be used later for (4.48).

We will allow for rather extreme loadings, satisfying only

$$
\begin{array}{ll}
f \in L^{1}\left(I ; L^{2}\left(\Omega ; \mathbb{R}^{d}\right)\right), \quad g \in L^{1}(Q), & h \in L^{1}(\Sigma), \\
u_{0} \in W^{1,2}\left(\Omega ; \mathbb{R}^{d}\right), \quad v_{0} \in L^{2}\left(\Omega ; \mathbb{R}^{d}\right), & \theta_{0} \in L^{\omega}(\Omega) .
\end{array}
$$

We will assume nonnegativity of the external heat sources:

$$
g \geq 0, \quad h \geq 0, \quad \theta_{0} \geq 0 .
$$

In the isotropic case in Example 2.2, from (2.12) we can see that (3.3a) is satisfied with $\eta_{0}=\mu$ and (3.35,e) holds with $\eta_{1}=\mu^{1}=\eta_{2}$, and (3.3e) needs $C_{0}=\max \left(\lambda^{0}+\right.$ $\left.2 \mu^{0}, \lambda^{1}+2 \mu^{1}\right)$.

Let us still abbreviate $I:=(0, T)$. We use the standard notation $C^{\infty}(\cdot)$ for the space of smooth (possibly vector- or matrix-valued) functions, $L^{p}(\cdot)$ for $p$-power Lebesgue integrable functions as well as $W^{k, p}(\cdot)$ for the Sobolev spaces of functions whose $k$ th derivatives are in $L^{p}(\cdot)$ on the domain indicated. If values range over a Banach space $X$, then $L^{p}(I ; X)$ refers to an $L^{p}$-Bochner space of Banach-space-valued functions while $W^{k, p}(I ; X)$ is a respective Sobolev-Bochner space. Moreover, $\mathcal{M}(\bar{I} ; X)$ denotes the space of $X$-valued measures on $\bar{I}=[0, T]$. Further, $(\cdot)^{*}$ will denote standardly the (topological) dual space. We also use ":" for the product of matrices and "." for the product of vectors. After transforming $\mathfrak{c}(\theta) \frac{\partial}{\partial t} \theta=\frac{\partial}{\partial t} \widehat{\mathfrak{c}}(\theta)$ in (2.2) with a primitive function $\widehat{\mathfrak{c}}$ of $\mathfrak{c}$, and by testing (2.1) by $z$ and thus transforming (2.2) by $w$ and using Green's formula and integration by parts in time and the boundary and initial conditions (2.3), we arrive at the following: 
Definition 3.1 (A very weak formulation). We will call $(u, \theta)$ with

$$
\begin{aligned}
& u \in L^{\infty}\left(I ; W^{1,2}\left(\Omega ; \mathbb{R}^{d}\right)\right) \cap W^{1, p}\left(I ; W^{1, p}\left(\Omega ; \mathbb{R}^{d}\right)\right) \cap W^{1, \infty}\left(I ; L^{2}\left(\Omega ; \mathbb{R}^{d}\right)\right), \\
& \theta \in L^{r}\left(I ; W^{1, r}(\Omega)\right) \cap L^{\infty}\left(I ; L^{\omega}(\Omega)\right) \quad \text { with any } \quad 1 \leq r<\frac{d+2 \omega}{d+\omega}
\end{aligned}
$$

a very weak solution to the thermo-visco-elastic system (2.1)-(2.2) with the boundary and initial conditions (2.3) if $u(0, \cdot)=u_{0}$ and if

$$
\begin{aligned}
\int_{Q}\left(\mathbb{C}(e(u)-\theta \mathbb{B})+\mathbb{D}\left(\frac{\partial e(u)}{\partial t}\right)\right) & : e(z)-\varrho \frac{\partial u}{\partial t} \cdot \frac{\partial z}{\partial t} \mathrm{~d} x \mathrm{~d} t \\
& =\int_{Q} f \cdot z \mathrm{~d} x \mathrm{~d} t+\int_{\Omega} \varrho v_{0}(x) \cdot z(0, x) \mathrm{d} x
\end{aligned}
$$

for any $z \in C^{\infty}\left(Q ; \mathbb{R}^{d}\right)$ with $z(T, \cdot)=0$, and if

$$
\begin{array}{r}
\int_{Q}\left(\mathbb{K} \nabla \theta \cdot \nabla w+\left(\theta \mathbb{C B}: \frac{\partial e(u)}{\partial t}-\mathbb{D}\left(\frac{\partial e(u)}{\partial t}\right): \frac{\partial e(u)}{\partial t}\right) w-\widehat{\mathfrak{c}}(\theta) \frac{\partial w}{\partial t}\right) \mathrm{d} x \mathrm{~d} t \\
=\int_{Q} g w \mathrm{~d} x \mathrm{~d} t+\int_{\Sigma} h w \mathrm{~d} S \mathrm{~d} t+\int_{\Omega} \widehat{\mathfrak{c}}\left(\theta_{0}(x)\right) w(0, x) \mathrm{d} x
\end{array}
$$

for any $w \in C^{\infty}(Q)$ with $w(T, \cdot)=0$, where $\widehat{\mathfrak{c}}$ denotes a primitive function of $\mathfrak{c}$.

Our main result is:

THEOREM 3.2 (Existence of very weak solutions). Under the assumptions (3.1)(3.5), a very weak solution to the thermo-visco-elastic system (2.1)-(2.2) with the boundary and initial conditions (2.3) does exist. In addition to (3.6), it also follows that

$$
\begin{aligned}
& \frac{\partial^{2} u}{\partial t^{2}} \in L^{p /(p-1)}\left(I ; W^{1, p}\left(\Omega ; \mathbb{R}^{d}\right)^{*}\right)+L^{1}\left(I ; L^{2}\left(\Omega ; \mathbb{R}^{d}\right)\right), \\
& \frac{\partial \theta}{\partial t} \in \mathcal{M}\left(I ; W^{-1-d / 2,2}(\Omega)\right), \\
& \frac{\partial \widetilde{\mathfrak{c}}(\theta)}{\partial t} \in L^{1}\left(I ; W^{-1-d / 2,2}(\Omega)\right) .
\end{aligned}
$$

Outline of proof. It follows from the arguments presented in Section 4, namely from Proposition 4.6. It is important that (3.4) allows both for (4.2) and for (4.3) used in Section 4 , while (3.5) allows for (4.20) below. As to (3.9k), it is important that $\frac{\partial}{\partial t} \widehat{\mathfrak{c}}(\theta)$ is not a measure in time but indeed belongs to $L^{1}\left(I ; W^{-1-d / 2,2}(\Omega)\right)$ because, in the sense of distributions, $\mathfrak{c}(\theta) \frac{\partial \theta}{\partial t}=\operatorname{div}(\mathbb{K} \nabla \theta)+\mathbb{D}\left(e\left(\frac{\partial u}{\partial t}\right)\right): e\left(\frac{\partial u}{\partial t}\right)-\theta \mathbb{C B}: e\left(\frac{\partial u}{\partial t}\right)+g \in$ $L^{1}\left(I ; W^{-1-d / 2,2}(\Omega)\right)$.

4. Analysis of the thermo-visco-elastic system. We will prove Theorem 3.2 by a careful successive passage to a limit in a suitably regularized Galerkin approximation and sophisticated $L^{1}$-type a priori estimates for the temperature gradient performed for the coupled system.

Considering a parameter $k \in \mathbb{N}$, one can always take a sequence of finite-dimensional subspaces $V_{k} \subset W^{1, \infty}(\Omega)$ that is increasing and approximates all of $W^{1, p}(\Omega)$ strongly; 
i.e.,

$$
V_{k} \subset V_{k+1} \quad \text { and } \quad \operatorname{cl}\left(\bigcup_{k \in \mathbb{N}} V_{k}\right)=W^{1, p}(\Omega),
$$

where "cl" refers to the closure in $W^{1, p}(\Omega)$. Moreover, we consider approximations $f_{k}$ of $f \in L^{1}\left(I ; L^{2}\left(\Omega ; \mathbb{R}^{d}\right)\right)$, and $g_{k}$ of $g \in L^{1}(Q)$, and also $h_{k}$ of $h \in L^{1}(\Sigma)$ such that

$$
\begin{array}{llll}
f_{k} \in L^{\infty}\left(Q ; \mathbb{R}^{d}\right) & \text { and } \quad \lim _{k \rightarrow \infty} f_{k}=f & \text { strongly in } L^{1}\left(I ; L^{2}\left(\Omega ; \mathbb{R}^{d}\right)\right), \\
g_{k} \in L^{2}(Q) & \text { and } \quad \lim _{k \rightarrow \infty} g_{k}=g & \text { strongly in } L^{1}(Q), \\
h_{k} \in W^{1,2}\left(I ; L^{2}(\Gamma)\right) & \text { and } \quad \lim _{k \rightarrow \infty} h_{k}=h & \text { strongly in } L^{1}(\Sigma) .
\end{array}
$$

We also approximate the initial conditions by some $u_{0, k}, v_{0, k}$ and $\theta_{0, k}$, and assume

$$
\begin{aligned}
& u_{0, k} \in V_{k}^{d} \quad \text { and } \quad \lim _{k \rightarrow \infty} u_{0, k}=u_{0} \quad \text { in } W^{1,2}\left(\Omega ; \mathbb{R}^{d}\right) \text {, } \\
& v_{0, k} \in V_{k}^{d} \quad \text { and } \quad \lim _{k \rightarrow \infty} v_{0, k}=v_{0} \quad \text { in } L^{2}\left(\Omega ; \mathbb{R}^{d}\right) \text {, } \\
& \theta_{0, k} \in V_{k} \quad \text { and } \quad \lim _{k \rightarrow \infty} \theta_{0, k}=\theta_{0} \quad \text { in } L^{\omega}(\Omega) .
\end{aligned}
$$

Moreover, we make a regularization of both the mechanical and the thermal parts by monotone terms with a sufficiently fast growth to compensate the growth of the righthand-side terms in the heat equation (2.2) just to guarantee mere existence of the Galerkin approximants. Later these terms will be suppressed.

Thus, for a regularization parameter $\varepsilon>0$, we define the Galerkin approximation $\left(u_{k l \varepsilon}, \theta_{k l \varepsilon}\right)$ of the regularized system as functions

$$
\begin{aligned}
& u_{k l \varepsilon} \in W^{1, \infty}\left(I ; V_{k}^{d}\right) \cap W^{2,1}\left(I ;\left(V_{k}^{d}\right)^{*}\right), \\
& \theta_{k l \varepsilon} \in L^{\infty}\left(I ; V_{l}\right) \cap W^{1,1}\left(I ; V_{l}^{*}\right)
\end{aligned}
$$

satisfying the initial conditions

$$
u_{k l \varepsilon}(0, \cdot)=u_{0, k}, \quad \frac{\partial u_{k l \varepsilon}}{\partial t}(0, \cdot)=v_{0, k}, \quad \theta_{k l \varepsilon}(0, \cdot)=\theta_{0, k}
$$

and

$$
\begin{array}{r}
\left\langle\varrho \frac{\partial^{2} u_{k l \varepsilon}}{\partial t^{2}}, z\right\rangle+\int_{\Omega}\left(\mathbb{C}\left(e\left(u_{k l \varepsilon}\right)-\theta_{k l \varepsilon} \mathbb{B}\right)+\mathbb{D}\left(\frac{\partial e\left(u_{k l \varepsilon}\right)}{\partial t}\right)\right): e(z) \\
+\varepsilon\left|\frac{\partial u_{k l \varepsilon}}{\partial t}\right|^{2 p-2} \frac{\partial u_{k l \varepsilon}}{\partial t} \cdot z \mathrm{~d} x=\int_{\Omega} f_{k} \cdot z \mathrm{~d} x
\end{array}
$$

for any $z \in V_{k}^{d}$ and for a.a. $t \in[0, T]$, and if

$$
\begin{gathered}
\left\langle\mathfrak{c}\left(\theta_{k l \varepsilon}\right) \frac{\partial \theta_{k l \varepsilon}}{\partial t}, w\right\rangle+\int_{\Omega}\left(\mathbb{K} \nabla \theta_{k l \varepsilon} \cdot \nabla w+\left(\varepsilon\left|\theta_{k l \varepsilon}\right| \theta_{k l \varepsilon}+\theta_{k l \varepsilon} \mathbb{C B}: \frac{\partial e\left(u_{k l \varepsilon}\right)}{\partial t}\right.\right. \\
\left.\left.-\mathbb{D}\left(\frac{\partial e\left(u_{k l \varepsilon}\right)}{\partial t}\right): \frac{\partial e\left(u_{k l \varepsilon}\right)}{\partial t}\right) w\right) \mathrm{d} x=\int_{\Omega} g_{k} w \mathrm{~d} x+\int_{\Gamma} h_{k} w \mathrm{~d} S
\end{gathered}
$$


for any $w \in V_{l}$ and for a.a. $t \in[0, T]$. In other words, $\left(u_{k l \varepsilon}, \theta_{k l \varepsilon}\right)$ solves, in the weak sense, the system

$$
\begin{aligned}
& \varrho \frac{\partial^{2} u_{k l \varepsilon}}{\partial t^{2}}-\operatorname{div}\left(\mathbb{C}\left(e\left(u_{k l \varepsilon}\right)-\theta_{k l \varepsilon} \mathbb{B}\right)+\mathbb{D}\left(\frac{\partial e\left(u_{k l \varepsilon}\right)}{\partial t}\right)\right) \\
& +\varepsilon\left|\frac{\partial u_{k l \varepsilon}}{\partial t}\right|^{2 p-2} \frac{\partial u_{k l \varepsilon}}{\partial t}=f_{k}+r_{k l \varepsilon}^{(1)} \\
& \left(\mathfrak{c}\left(\theta_{k l \varepsilon}\right) \frac{\partial \theta_{k l \varepsilon}}{\partial t}-\operatorname{div}\left(\mathbb{K} \nabla \theta_{k l \varepsilon}\right)+\varepsilon\left|\theta_{k l \varepsilon}\right| \theta_{k l \varepsilon}+\theta_{k l \varepsilon} \mathbb{C B}: \frac{\partial e\left(u_{k l \varepsilon}\right)}{\partial t}\right. \\
& \left.-\mathbb{D}\left(\frac{\partial e\left(u_{k l \varepsilon}\right)}{\partial t}\right): \frac{\partial e\left(u_{k l \varepsilon}\right)}{\partial t}-g_{k}, \mathbb{K} \frac{\partial \theta_{k l \varepsilon}}{\partial \nu}-h_{k}\right)=r_{k l \varepsilon}^{(2)},
\end{aligned}
$$

where the residua $r_{k l \varepsilon}^{(1)} \in W^{1, p}\left(\Omega ; \mathbb{R}^{d}\right)^{*}$ and $r_{k l \varepsilon}^{(2)} \in W^{1,2}(\Omega)^{*}$ are orthogonal to $V_{k}^{d}$ and $V_{l}$, respectively. In the following proofs, we can confine ourselves on $k \geq l$ so that, in particular, $\theta_{0, k} \in V_{k} \subset V_{l}$. The meaning of the left-hand side of (4.8b), let us denote it by $\left(\xi_{k l \varepsilon}, \zeta_{k l \varepsilon}\right)$, is naturally the (continuous extension of the) linear functional $z \mapsto$ $\int_{\Omega} \xi_{k l \varepsilon} z \mathrm{~d} x+\int_{\Gamma} \zeta_{k l \varepsilon} z \mathrm{~d} S$.

Let us further define the seminorms on a dual space $\mathcal{V}^{*}$ to a function space $\mathcal{V} \subset$ $L^{1}\left(Q ; \mathbb{R}^{n}\right)$ for $n:=d$ or $n:=1$ by

$$
|f|_{k, \mathcal{V}^{*}}:=\sup _{\substack{\|v\|_{\mathcal{\nu}} \leq 1 \\ v(t, \cdot) \in V_{k}^{n} \text { for a.a. } t \in[0, T]}} \int_{Q} f \cdot v \mathrm{~d} x \mathrm{~d} t .
$$

Considering a (countable) collection of these seminorms for $k \in \mathbb{N}$, we generate a locally convex topology on $\mathcal{V}^{*}$. Equipped with such a topology, this locally convex space will then be denoted by $\mathcal{V}_{\mathrm{LCS}}^{*}$; for this construction, see also [28, Sect.8.4]. Thanks to the assumption (4.1), these spaces are Hausdorff topological spaces.

Lemma 4.1 (Existence of Galerkin solution, a priori estimates). Let (3.1), (3.2), (3.3), (4.2) and (4.3) hold. Then the Galerkin approximate solution $\left(u_{k l \varepsilon}, \theta_{k l \varepsilon}\right)$ to the regularized system (4.4)-(4.7) does exist and satisfies, for some $C_{1 \ldots 4, k \varepsilon}<+\infty$ dependent on $k$ and $\varepsilon$ but not on $l$, the following a priori estimates:

$$
\begin{aligned}
& \left\|u_{k l \varepsilon}\right\|_{W^{1, \infty}\left(I ; W^{1, \infty}\left(\Omega ; \mathbb{R}^{d}\right)\right)} \leq C_{1, k \varepsilon}, \\
& \left|\frac{\partial^{2} u_{k l \varepsilon}}{\partial t^{2}}\right|_{k, L^{1}\left(I ; W^{1,2}\left(\Omega ; \mathbb{R}^{d}\right)\right)^{*}} \leq C_{2, k \varepsilon}, \\
& \left\|\theta_{k l \varepsilon}\right\|_{L^{\infty}\left(I ; L^{1+\omega}(\Omega)\right) \cap L^{2}\left(I ; W^{1,2}(\Omega)\right) \cap L^{3}(Q)} \leq C_{3, k \varepsilon}, \\
& \left|\frac{\partial \theta_{k l \varepsilon}}{\partial t}\right|_{\widehat{l},\left(L^{2}\left(I ; W^{1,2}(\Omega)\right) \cap L^{3}(Q)\right)^{*}} \leq C_{4, k \varepsilon} \quad \text { provided } l \geq \widehat{l} .
\end{aligned}
$$

Proof. First, the existence of the Galerkin solution follows by standard arguments based on the theory of ordinary differential equations together with a successive prolongation which is made possible due to the $L^{\infty}(I ; \cdot)$-estimates of $u_{k l \varepsilon}$ and of $\theta_{k l \varepsilon}$ derived below.

We perform the test of (4.6) and of (4.7) by $z:=\frac{\partial}{\partial t} u_{k l \varepsilon}(t, \cdot)$ and $w:=\theta_{k l \varepsilon}(t, \cdot)$, which are legal test functions being from $V_{k}^{d}$ and $V_{l}$, respectively. We will also use Korn's 
inequality in the form

$$
\begin{aligned}
& \exists \eta_{3}>0 \quad \forall v \in W^{1, p}\left(\Omega ; \mathbb{R}^{d}\right): \\
& \eta_{3}\|v\|_{W^{1, p}\left(\Omega ; \mathbb{R}^{d}\right)} \leq\|e(v)\|_{L^{p}\left(\Omega ; \mathbb{R}_{\mathrm{sym}}^{d \times d}\right)}+\|v\|_{L^{2}\left(\Omega ; \mathbb{R}^{d}\right)}
\end{aligned}
$$

with $\eta_{3}>0$ depending on $\Omega$, relying on (3.1). From (3.3d), combined with (4.11) and the algebraic inequality $(a-b)^{p} \geq 2^{1-p} a^{p}-b^{p}$, we obtain

$$
\begin{aligned}
\int_{\Omega} \mathbb{D} & \left(\frac{\partial e\left(u_{k l \varepsilon}\right)}{\partial t}\right): \frac{\partial e\left(u_{k l \varepsilon}\right)}{\partial t} \mathrm{~d} x \geq \eta_{1}\left\|\frac{\partial e\left(u_{k l \varepsilon}\right)}{\partial t}\right\|_{L^{p}\left(\Omega ; \mathbb{R}_{\mathrm{sym}}^{d \times d}\right)}^{p} \\
& \geq \eta_{1}\left(\eta_{3}\left\|\frac{\partial u_{k l \varepsilon}}{\partial t}\right\|_{W^{1, p}\left(\Omega ; \mathbb{R}^{d}\right)}-\left\|\frac{\partial u_{k l \varepsilon}}{\partial t}\right\|_{L^{2}\left(\Omega ; \mathbb{R}^{d}\right)}\right)^{p} \\
& \geq 2^{1-p} \eta_{1} \eta_{3}^{p}\left\|\frac{\partial u_{k l \varepsilon}}{\partial t}\right\|_{W^{1, p}\left(\Omega ; \mathbb{R}^{d}\right)}^{p}-\eta_{1}\left\|\frac{\partial u_{k l \varepsilon}}{\partial t}\right\|_{L^{2}\left(\Omega ; \mathbb{R}^{d}\right)}^{p}
\end{aligned}
$$

We also introduce $\mathfrak{C}: \mathbb{R} \rightarrow \mathbb{R}$ defined by $\mathfrak{C}(\theta):=\int_{0}^{\theta} \vartheta \mathfrak{c}(\vartheta) \mathrm{d} \vartheta$. As we do not have nonnegativity of $\theta_{k l \varepsilon}$ guaranteed, we can formally define $\mathfrak{c}(\theta)=\mathfrak{c}(-\theta)$ for $\theta<0$. Then (3.3. $\left.\mathrm{h}\right)$ ensures $\frac{1}{1+\omega} c_{\min }|\theta|^{1+\omega} \leq \mathfrak{C}(\theta) \leq c_{\max }\left(\frac{1}{2} \theta^{2}+\frac{1}{1+\omega}|\theta|^{1+\omega}\right)$. Hence, these tests yield in the sum:

$$
\begin{aligned}
& \frac{1}{2} \frac{\mathrm{d}}{\mathrm{d} t}\left(\varrho\left\|\frac{\partial u_{k l \varepsilon}}{\partial t}\right\|_{L^{2}\left(\Omega ; \mathbb{R}^{d}\right)}^{2}+\int_{\Omega} \mathbb{C} e\left(u_{k l \varepsilon}\right): e\left(u_{k l \varepsilon}\right)+\mathfrak{C}\left(\theta_{k l \varepsilon}\right) \mathrm{d} x\right) \\
& \quad+2^{1-p} \eta_{1} \eta_{3}^{p}\left\|\frac{\partial u_{k l \varepsilon}}{\partial t}\right\|_{W^{1, p}\left(\Omega ; \mathbb{R}^{d}\right)}^{p}-\eta_{1}\left\|\frac{\partial u_{k l \varepsilon}}{\partial t}\right\|_{L^{2}\left(\Omega ; \mathbb{R}^{d}\right)}^{p}+\kappa\left\|\nabla \theta_{k l \varepsilon}\right\|_{L^{2}\left(\Omega ; \mathbb{R}^{d}\right)}^{2} \\
& \quad+\varepsilon\left(\left\|\frac{\partial u_{k l \varepsilon}}{\partial t}\right\|_{L^{2 p}\left(\Omega ; \mathbb{R}^{d}\right)}^{2 p}+\left\|\theta_{k l \varepsilon}\right\|_{L^{3}(\Omega)}^{3}\right) \\
& \leq \int_{\Omega}\left(\varrho \frac{\partial^{2} u_{k l \varepsilon}}{\partial t^{2}}+\varepsilon\left|\frac{\partial u_{k l \varepsilon}}{\partial t}\right|^{2 p-2} \frac{\partial u_{k l \varepsilon}}{\partial t}\right) \cdot \frac{\partial u_{k l \varepsilon}}{\partial t}+\left(\mathbb{C} e\left(u_{k l \varepsilon}\right)+\mathbb{D}\left(\frac{\partial e\left(u_{k l \varepsilon}\right)}{\partial t}\right)\right) \\
& \quad: e\left(\frac{\partial u_{k l \varepsilon}}{\partial t}\right)+\left(\mathfrak{c}\left(\theta_{k l \varepsilon}\right) \frac{\partial \theta_{k l \varepsilon}}{\partial t}+\varepsilon\left|\theta_{k l \varepsilon}\right| \theta_{k l \varepsilon}\right) \theta_{k l \varepsilon}+\mathbb{K} \nabla \theta_{k l \varepsilon} \cdot \nabla \theta_{k l \varepsilon} \\
& =\int_{\Omega} \mathbb{D}\left(\frac{\partial e\left(u_{k l \varepsilon}\right)}{\partial t}\right): \frac{\partial e\left(u_{k l \varepsilon}\right)}{\partial t} \theta_{k l \varepsilon}-\mathbb{C B}: \frac{\partial e\left(u_{k l \varepsilon}\right)}{\partial t} \theta_{k l \varepsilon}^{2} \\
& \quad+f_{k}(t, \cdot) \cdot \frac{\partial u_{k l \varepsilon}}{\partial t}+g_{k}(t, \cdot) \theta_{k l \varepsilon} \mathrm{d} x+\int_{\Gamma} h_{k}(t, \cdot) \theta_{k l \varepsilon} \mathrm{d} S .
\end{aligned}
$$

By (3.3e and by using Hölder's and Young's inequalities, we have

$$
\begin{aligned}
\int_{\Omega} \mathbb{C B}: \frac{\partial e\left(u_{k l \varepsilon}\right)}{\partial t} \theta_{k l \varepsilon}^{2} & \leq|\mathbb{C B}| \int_{\Omega}\left|\frac{\partial e\left(u_{k l \varepsilon}\right)}{\partial t}\right|\left|\theta_{k l \varepsilon}\right|^{2} \mathrm{~d} x \\
& \leq C_{\varepsilon, k, p}+\frac{\varepsilon}{4}\left\|\frac{u_{k l \varepsilon}}{\partial t}\right\|_{L^{2 p}\left(\Omega ; \mathbb{R}^{d}\right)}^{2 p}+\frac{\varepsilon}{2}\left\|\theta_{k l \varepsilon}\right\|_{L^{3}(\Omega)}^{3}
\end{aligned}
$$

where the constant $C_{\varepsilon, k, p}$ depends on its indices and on $\mathbb{B}$ and $\mathbb{C}$; we used also that always $2 p \geq 3$ (here (3.2) is used) and that the $W^{1,2 p}(\Omega)$ - and $L^{2 p}(\Omega)$-norms are equivalent to 
each other if restricted on $V_{k}$ with $k$ fixed. Similarly,

$$
\begin{aligned}
\int_{\Omega} \mathbb{D}\left(\frac{\partial e\left(u_{k l \varepsilon}\right)}{\partial t}\right): & \frac{\partial e\left(u_{k l \varepsilon}\right)}{\partial t} \theta_{k l \varepsilon} \mathrm{d} x \\
& \leq \int_{\Omega} C_{0}\left(1+\left|\frac{\partial e\left(u_{k l \varepsilon}\right)}{\partial t}\right|^{p-1}\right)\left|\frac{\partial e\left(u_{k l \varepsilon}\right)}{\partial t}\right|\left|\theta_{k l \varepsilon}\right| \mathrm{d} x \\
& \leq \int_{\Omega} \delta\left(1+\left|\frac{\partial e\left(u_{k l \varepsilon}\right)}{\partial t}\right|^{2 p}\right)+\frac{2 C_{0}^{2}}{\delta}\left|\theta_{k l \varepsilon}\right|^{2} \mathrm{~d} x \\
& \leq C_{\varepsilon, k, p}\left(1+\left\|\theta_{k l \varepsilon}\right\|_{L^{2}(\Omega)}^{2}\right)+\frac{\varepsilon}{4}\left\|\frac{\partial u_{k l \varepsilon}}{\partial t}\right\|_{L^{2 p}\left(\Omega ; \mathbb{R}^{d}\right)}^{2 p}
\end{aligned}
$$

where $\delta>0$ was suitably chosen to give the constant $C_{\varepsilon, k, p}$ now depending also on $C_{0}$. The remaining terms on the right-hand side of (4.13) can be estimated even more easily. Eventually, we estimate the nonpositive term on the left-hand side of (4.13) as

$$
\eta_{1}\left\|\frac{\partial u_{k l \varepsilon}}{\partial t}\right\|_{L^{2}\left(\Omega ; \mathbb{R}^{d}\right)}^{p} \leq \eta_{1}|\Omega|^{p / 2}\left\|\frac{\partial u_{k l \varepsilon}}{\partial t}\right\|_{L^{2 p}\left(\Omega ; \mathbb{R}^{d}\right)}^{p} \leq C_{\varepsilon, p,|\Omega|, \eta_{1}}+\frac{\varepsilon}{4}\left\|\frac{\partial u_{k l \varepsilon}}{\partial t}\right\|_{L^{2 p}\left(\Omega ; \mathbb{R}^{d}\right)}^{2 p},
$$

where the constant $C_{\varepsilon, p,|\Omega|, \eta_{1}}$ depends again on its indices. Then we absorb the $\varepsilon$-terms in these estimates in the left-hand side of (4.13). Further we use also Gronwall's inequality, which eventually yields (4.10c) and the estimates of $u_{k l \varepsilon}$ in $W^{1, \infty}\left(I ; L^{2}\left(\Omega ; \mathbb{R}^{d}\right)\right)$ and $L^{\infty}\left(I ; W^{1,2}\left(\Omega ; \mathbb{R}^{d}\right)\right)$. From this, the estimate 4.10a) then directly follows because $V_{k} \subset W^{1, \infty}\left(\Omega ; \mathbb{R}^{d}\right)$ is assumed and the $L^{2}$-norm on the finite-dimensional subspace $V_{k}$ is equivalent to the norm induced from $W^{1, \infty}\left(\Omega ; \mathbb{R}^{d}\right)$; of course, again this estimate cannot be uniform with respect to $k$.

The estimate (4.10b) can be obtained by a test of (4.6) by $z(t, \cdot) \in V_{k}^{d}$ with $z \in$ $L^{1}\left(I ; W^{1,2}\left(\Omega ; \mathbb{R}^{d}\right)\right)$, performing an integration over $I$, and estimating the supremum as suggested in (4.9) routinely by using Hölder's inequality and the already proved estimates (4.10h,c). E.g. we estimate

$$
\begin{aligned}
\int_{Q} \theta_{k l \varepsilon} \mathbb{C B}: e(z) \mathrm{d} x \mathrm{~d} t & \leq|\mathbb{C B}|\left\|\theta_{k l \varepsilon}\right\|_{L^{\infty}\left(I ; L^{2}(\Omega)\right)}\|e(z)\|_{L^{1}\left(I ; L^{2}\left(\Omega ; \mathbb{R}^{d \times d}\right)\right)} \\
& \leq|\mathbb{C B}| C_{3, k \varepsilon}\|z\|_{L^{1}\left(I ; W^{1,2}\left(\Omega ; \mathbb{R}^{d}\right)\right)},
\end{aligned}
$$

the other details being omitted. Similarly, 4.10d can be obtained by a test of (4.7) by $z(t, \cdot) \in V_{l}$ with $z \in L^{2}\left(I ; W^{1,2}(\Omega)\right) \cap L^{3}(Q)$.

Now, realizing that the a priori estimates (4.10) are independent of $l$, we pass to the limit with $l \rightarrow \infty$, obtaining thus a solution $\left(\theta_{k \varepsilon}, u_{k \varepsilon}\right)$ to a "semi-Galerkin" approximation involving the discretized and regularized mechanical part and the continuous (but still regularized) heat equation that can thus be tested by various nonlinear functions of $\theta$.

Lemma 4.2 (Limit passage for $l \rightarrow \infty$ ). Let the assumptions of Lemma 4.1 together with (4.1) hold. Then the sequence $\left\{\left(u_{k l \varepsilon}, \theta_{k l \varepsilon}\right)\right\}_{l \in \mathbb{N}}$ contains a subsequence converging weakly* in the (locally convex) topologies indicated by the estimates (4.10) to some limit; let us denote it by $\left(u_{k \varepsilon}, \theta_{k \varepsilon}\right)$. Each $u_{k \varepsilon} \in W^{1,2}\left(I ; V_{k}^{d}\right)$ and $\theta_{k \varepsilon} \in L^{2}\left(I ; W^{1,2}(\Omega)\right)$ thus 
obtained solves, in the weak sense, the system

$$
\begin{aligned}
& \varrho \frac{\partial^{2} u_{k \varepsilon}}{\partial t^{2}}-\operatorname{div}\left(\mathbb{C}\left(e\left(u_{k \varepsilon}\right)-\theta_{k \varepsilon} \mathbb{B}\right)+\mathbb{D}\left(\frac{\partial e\left(u_{k \varepsilon}\right)}{\partial t}\right)\right) \\
& +\varepsilon\left|\frac{\partial u_{k \varepsilon}}{\partial t}\right|^{2 p-2} \frac{\partial u_{k \varepsilon}}{\partial t}=f_{k}+r_{k \varepsilon}, \\
& \mathfrak{c}\left(\theta_{k \varepsilon}\right) \frac{\partial \theta_{k \varepsilon}}{\partial t}-\operatorname{div}\left(\mathbb{K} \nabla \theta_{k \varepsilon}\right)+\varepsilon\left|\theta_{k \varepsilon}\right| \theta_{k \varepsilon} \\
& =\mathbb{D}\left(\frac{\partial e\left(u_{k \varepsilon}\right)}{\partial t}\right): \frac{\partial e\left(u_{k \varepsilon}\right)}{\partial t}-\theta_{k \varepsilon} \mathbb{C B}: \frac{\partial e\left(u_{k \varepsilon}\right)}{\partial t}+g_{k},
\end{aligned}
$$

with the initial conditions $u_{k \varepsilon}(0, \cdot)=u_{0, k}, \frac{\partial u_{k \varepsilon}}{\partial t}(0, \cdot)=v_{0, k}$, and $\theta_{k \varepsilon}(0, \cdot)=\theta_{0, k}$ and the boundary conditions $\sigma_{k \varepsilon} \nu=0$ and $\mathbb{K} \frac{\partial}{\partial \nu} \theta_{k \varepsilon}=h_{k}$ on $\Sigma$ with $\sigma_{k \varepsilon}$ denoting the stress tensor as in $(2.1 \mathrm{~b})$ but with $u_{k \varepsilon}$ in place of $u$, and where the residuum $r_{k \varepsilon} \in W^{1, p}\left(\Omega ; \mathbb{R}^{d}\right)^{*}$ is orthogonal to $V_{k}^{d}$. Moreover, (4.10) is inherited by this limit in the sense

$$
\begin{aligned}
& \left\|u_{k \varepsilon}\right\|_{W^{1, \infty}\left(I ; W^{1, \infty}\left(\Omega ; \mathbb{R}^{d}\right)\right)} \leq C_{1, k \varepsilon}, \\
& \left|\frac{\partial^{2} u_{k \varepsilon}}{\partial t^{2}}\right|_{k, L^{1}\left(I ; W^{1,2}\left(\Omega ; \mathbb{R}^{d}\right)\right)^{*}} \leq C_{2, k \varepsilon}, \\
& \left\|\theta_{k \varepsilon}\right\|_{L^{\infty}\left(I ; L^{1+\omega}(\Omega)\right) \cap L^{2}\left(I ; W^{1,2}(\Omega)\right) \cap L^{3}(Q)} \leq C_{3, k \varepsilon}, \\
& \left\|\frac{\partial \theta_{k \varepsilon}}{\partial t}\right\|_{\left(L^{2}\left(I ; W^{1,2}(\Omega)\right) \cap L^{3}(Q)\right)^{*}} \leq C_{4, k \varepsilon} \text {. }
\end{aligned}
$$

Proof. Take a subsequence $u_{k l \varepsilon} \stackrel{*}{\rightarrow} u_{k \varepsilon}$ and $\theta_{k l \varepsilon} \stackrel{*}{\rightarrow} \theta_{k \varepsilon}$ as announced in this proposition. The a priori estimates (4.18) are inherited from (4.10). The fact that $\frac{\partial \theta_{k \varepsilon}}{\partial t} \in$ $\left(L^{2}\left(I ; W^{1,2}(\Omega)\right) \cap L^{3}(Q)\right)^{*}$ can be obtained by a successive extension of a limit of a subsequence of functionals $\frac{\partial \theta_{k l \varepsilon}}{\partial t}$ from $L^{2}\left(I ; V_{l}\right)$ onto $L^{2}\left(I ; V_{l+1}\right)$ and then, by continuity, on all of $L^{2}\left(I ; W^{1,2}(\Omega)\right) \cap L^{3}(Q)$; cf. [28, the proof of Theorem 8.27]. For this, it is important that (4.10d) is uniform with respect to $l$. The estimate (4.18d) is then inherited from (4.10d).

By Aubin-Lions' compact-embedding theorem [1, 17] generalized for the locally-convex spaces together with the fact that $V_{k}^{d}$ is finite-dimensional (hence compactly embedded into itself) and with an interpolation by the Gagliardo-Nirenberg inequality, cf. [28, Lemmas 7.7-7.8], the estimates (4.10, a,c-e) imply that

$$
\begin{aligned}
& \frac{\partial u_{k l \varepsilon}}{\partial t} \rightarrow \frac{\partial u_{k \varepsilon}}{\partial t} \text { in } L^{q}\left(I ; V_{k}^{d}\right), \\
& \theta_{k l \varepsilon} \rightarrow \theta_{k \varepsilon} \quad \text { in } \quad L^{q_{1}}(Q)
\end{aligned}
$$

with any $q<+\infty$ and $q_{1}<3$. The proof that the weak limit $\left(u_{k \varepsilon}, \theta_{k \varepsilon}\right)$ is the sought weak solution to (4.17) is then simple. Indeed, having the strong convergence $\frac{\partial}{\partial t} e\left(u_{k l \varepsilon}\right) \rightarrow$ $\frac{\partial}{\partial t} e\left(u_{k \varepsilon}\right)$ in $L^{q}\left(I ; L^{\infty}\left(\Omega ; \mathbb{R}_{\mathrm{sym}}^{d \times d}\right)\right)$, see $(4.19 \mathrm{a})$, and hence surely in $L^{p}\left(Q ; \mathbb{R}_{\mathrm{sym}}^{d \times d}\right)$, we can pass to the limit in the term $\mathbb{D}\left(\frac{\partial}{\partial t} e\left(u_{k l \varepsilon}\right)\right): \frac{\partial}{\partial t} e\left(u_{k l \varepsilon}\right) \rightarrow \mathbb{D}\left(\frac{\partial}{\partial t} e\left(u_{k \varepsilon}\right)\right): \frac{\partial}{\partial t} e\left(u_{k \varepsilon}\right)$ in $L^{1}(Q)$ in the Galerkin identity (4.7). The other nonlinear terms in the heat equation (4.8b) are even simpler. We can consider $w$ in (4.7) as $w_{l} \in C^{1}\left(I ; V_{l}\right)$ converging strongly to $w$ in $C^{1}\left(I ; W^{1,2}(\Omega)\right)$ and, after making the limit passage with $l \rightarrow \infty$, we obtain directly the weak formulation of (4.17b) with the mentioned initial/boundary conditions. The strong 
convergences (4.19) also allow for a direct limit passage in the Galerkin identity (4.6) to get the Galerkin identity corresponding to (4.17).

Having $\theta_{k \varepsilon}$ "continuous" (i.e. not of the Galerkin type), we can use various "nonlinear" tests for an advanced estimation technique for $\nabla \theta_{k \varepsilon}$ developed, for the separate heat equation, by Boccardo and Gallouët [4] and later augmented in [3]. Here, we nontrivially combine this technique with an estimation of the mechanical part. This allows for optimal estimation, especially of the adiabatic "C্B-term". Simultaneously we can still benefit from having (4.18a) at our disposal. In view of (3.5) with (4.2 b,c) and (4.3b), it is consistent to assume

$$
g_{k} \geq 0, \quad h_{k} \geq 0, \quad u_{0, k} \geq 0 .
$$

Proposition 4.3 (Further estimates for $u_{k \varepsilon}$ and $\theta_{k \varepsilon}$ ). Let the assumptions of Lemma 4.1, (4.1) and now also (4.20) hold. Then, for any $\delta>0, u_{k \varepsilon}$ and $\theta_{k \varepsilon}$ satisfy also

$$
\begin{aligned}
& \left\|u_{k \varepsilon}\right\|_{W^{1, \infty}\left(I ; L^{2}\left(\Omega ; \mathbb{R}^{d}\right)\right) \cap L^{\infty}\left(I ; W^{1,2}\left(\Omega ; \mathbb{R}^{d}\right)\right)} \leq C_{5}, \\
& \left\|\frac{\partial e\left(u_{k \varepsilon}\right)}{\partial t}\right\|_{\left.L^{p}\left(Q ; \mathbb{R}^{d \times d}\right)\right)} \leq C_{6}, \\
& \left|\frac{\partial^{2} u_{k \varepsilon}}{\partial t^{2}}\right|_{\widehat{k},\left(L^{p}\left(I ; W^{1, p}\left(\Omega ; \mathbb{R}^{d}\right)\right) \cap L^{\infty}\left(I ; L^{2}\left(\Omega ; \mathbb{R}^{d}\right)\right) \cap L^{2 p}\left(Q ; \mathbb{R}^{d}\right)\right)^{*}} \leq C_{7} \quad \text { for } k \geq \widehat{k}, \\
& \left\|\theta_{k \varepsilon}\right\|_{L^{\infty}\left(I ; L^{\omega}(\Omega)\right)} \leq C_{8}, \\
& \left\|\nabla \theta_{k \varepsilon}\right\|_{L^{r}\left(\Omega ; \mathbb{R}^{d}\right)} \leq C_{9, r}, \quad 1 \leq r<\frac{d+2 \omega}{d+\omega}, \\
& \left\|\frac{\partial \theta_{k \varepsilon}}{\partial t}\right\|_{L^{1}\left(I ; W^{-1-d / 2,2}(\Omega)\right)} \leq C_{10}
\end{aligned}
$$

with the $C$ 's independent of $k$ and $\varepsilon$. Moreover, $\theta_{k \varepsilon} \geq 0$, and also

$$
\begin{aligned}
& \left\|\frac{\partial u_{k \varepsilon}}{\partial t}\right\|_{L^{2 p}\left(Q ; \mathbb{R}^{d}\right)} \leq C_{11} \varepsilon^{-1 /(2 p)}, \\
& \left\|\theta_{k \varepsilon}\right\|_{L^{3}(Q)} \leq C_{12, k} \varepsilon^{-1 / 3}
\end{aligned}
$$

where again $C_{11}$ does not depend on $k$ and $\varepsilon$ but $C_{12, k}$ does depend on $k$ as indicated.

Proof. First, we prove $\theta_{k \varepsilon} \geq 0$ standardly by testing the heat equation by $\theta_{k \varepsilon}^{-}$. Here we use (4.20) as well as the fact that $\frac{\partial}{\partial t} \theta_{k \varepsilon}$ is in duality with $\theta_{k \varepsilon}$ and then also with $\theta_{k \varepsilon}^{-} \in L^{2}\left(I ; W^{1,2}(\Omega)\right)$; cf. (4.18k, d).

Having the information $\theta_{k \varepsilon} \geq 0$, the energy balance can be obtained by multiplication of (4.17a) by $\frac{\partial}{\partial t} u_{k \varepsilon}$ and (4.17b) by 1 , and by using Green's formula both for (2.1) and 
for (2.2):

$$
\begin{aligned}
& \frac{\mathrm{d}}{\mathrm{d} t}\left(\frac{\rho}{2}\left\|\frac{\partial u_{k \varepsilon}}{\partial t}\right\|_{L^{2}\left(\Omega ; \mathbb{R}^{d}\right)}^{2}+\int_{\Omega} \frac{1}{2} \mathbb{C} e\left(u_{k \varepsilon}\right): e\left(u_{k \varepsilon}\right)\right) \\
& \quad+\int_{\Omega}\left(\mathbb{D}\left(\frac{\partial e\left(u_{k \varepsilon}\right)}{\partial t}\right): \frac{\partial e\left(u_{k \varepsilon}\right)}{\partial t}+\theta_{k \varepsilon} \mathbb{C B}: \frac{\partial e\left(u_{k \varepsilon}\right)}{\partial t}+\varepsilon\left|\frac{\partial u_{k \varepsilon}}{\partial t}\right|^{2 p}\right) \mathrm{d} x \\
& =\int_{\Omega} f_{k} \cdot \frac{\partial u_{k \varepsilon}}{\partial t} \mathrm{~d} x, \\
& \frac{\mathrm{d}}{\mathrm{d} t} \int_{\Omega} \widehat{\mathfrak{c}}\left(\theta_{k \varepsilon}\right) \mathrm{d} x-\int_{\Omega}\left(\mathbb{D}\left(\frac{\partial e\left(u_{k \varepsilon}\right)}{\partial t}\right): \frac{\partial e\left(u_{k \varepsilon}\right)}{\partial t}\right. \\
& \left.\quad+\theta_{k \varepsilon} \mathbb{C B}: \frac{\partial e\left(u_{k \varepsilon}\right)}{\partial t}+\varepsilon\left|\theta_{k \varepsilon}\right| \theta_{k \varepsilon}\right) \mathrm{d} x=\int_{\Omega} g_{k} \mathrm{~d} x+\int_{\Gamma} h_{k} \mathrm{~d} S,
\end{aligned}
$$

where $\widehat{\mathfrak{c}}$ is a primitive function of $\mathfrak{c}$; choosing $\widehat{\mathfrak{c}}(\theta)=\int_{0}^{\theta} \mathfrak{c}(\cdot)$, by $(\underline{3.3 \mathrm{~h}})$ it follows that

$$
\hat{c}_{\min } \theta^{\omega} \leq \widehat{\mathfrak{c}}(\theta) \leq \hat{c}_{\max }\left(1+\theta^{\omega}\right)
$$

for some $0<\hat{c}_{\min } \leq \hat{c}_{\max }<+\infty$. Summing (4.23) with (4.24), we get the total-energy balance:

$$
\begin{array}{r}
\frac{\mathrm{d}}{\mathrm{d} t} \int_{\Omega}\left(\frac{\rho}{2}\left|\frac{\partial u_{k \varepsilon}}{\partial t}\right|^{2}+\frac{1}{2} \mathbb{C} e\left(u_{k \varepsilon}\right): e\left(u_{k \varepsilon}\right)+\widehat{\mathfrak{c}}\left(\theta_{k \varepsilon}\right)\right) \mathrm{d} x-\int_{\Omega} f_{k} \cdot \frac{\partial u_{k \varepsilon}}{\partial t} \mathrm{~d} x \\
-\int_{\Omega} g_{k} \mathrm{~d} x-\int_{\Gamma} h_{k} \mathrm{~d} S=-\varepsilon \int_{\Omega}\left(\left|\frac{\partial u_{k \varepsilon}}{\partial t}\right|^{2 p}+\left|\theta_{k \varepsilon}\right| \theta_{k \varepsilon}\right) \mathrm{d} x \leq 0 .
\end{array}
$$

Since $\theta_{k \varepsilon} \geq 0$, by estimating $\int_{\Omega} f_{k} \cdot \frac{\partial u_{k \varepsilon}}{\partial t} \mathrm{~d} x \leq\left\|f_{k}\right\|_{L^{2}\left(\Omega ; \mathbb{R}^{d}\right)}\left(1+\left\|\frac{\partial u_{k \varepsilon}}{\partial t}\right\|_{L^{2}\left(\Omega ; \mathbb{R}^{d}\right)}\right)$ and by using Gronwall's inequality and the assumption (4.3) with the growth (4.25) of $\widehat{\mathfrak{c}}$, the estimate (4.26) yields the $L^{\infty}$-estimates in (4.21), d) independently of $\varepsilon$ and $k$. Here we use also the coercivity (4.25) of $\widehat{\mathfrak{c}}$. Still, however, this does not allow for a limit passage in the nonlinear term $\theta_{k \varepsilon} \mathbb{C B}: \frac{\partial}{\partial t} e\left(u_{k \varepsilon}\right)$, nor in $\mathbb{D}\left(\frac{\partial}{\partial t} e\left(u_{k \varepsilon}\right)\right): \frac{\partial}{\partial t} e\left(u_{k \varepsilon}\right)$.

Following the technique from [3, 4], we test (2.2) by $\phi_{n}\left(\theta_{k \varepsilon}\right)$ with $\phi_{n}: \mathbb{R}^{+} \rightarrow[0,1]$ defined by

$$
\phi_{n}(\theta):= \begin{cases}0 & \text { if } \theta \leq n \\ \theta-n & \text { if } n \leq \theta \leq n+1 \\ 1 & \text { if } \theta \geq n+1\end{cases}
$$

Denote by $\widehat{\phi}_{n}$ the primitive function of $\phi_{n}$ such that $\widehat{\phi}_{n}(0)=0$ and

$$
R_{k \varepsilon}:=\mathbb{D}\left(\frac{\partial e\left(u_{k \varepsilon}\right)}{\partial t}\right): \frac{\partial e\left(u_{k \varepsilon}\right)}{\partial t}-\theta_{k \varepsilon} \mathbb{C B}: \frac{\partial e\left(u_{k \varepsilon}\right)}{\partial t} .
$$


By integration in time and the Green formula and denoting $B_{n}:=\{(t, x) \in Q: n \leq$ $\left.\theta_{k \varepsilon}(t, x) \leq n+1\right\}$, we get

$$
\begin{aligned}
& \kappa \int_{B_{n}}\left|\nabla \theta_{k \varepsilon}\right|^{2} \mathrm{~d} x \mathrm{~d} t=\kappa \int_{Q} \phi_{n}^{\prime}\left(\theta_{k \varepsilon}\right)\left|\nabla \theta_{k \varepsilon}\right|^{2} \mathrm{~d} x \mathrm{~d} t \\
& \leq \int_{Q}\left(\mathbb{K} \nabla \theta_{k \varepsilon} \cdot \nabla \theta_{k \varepsilon}\right) \phi_{n}^{\prime}\left(\theta_{k \varepsilon}\right) \mathrm{d} x \mathrm{~d} t=\int_{Q} \mathbb{K} \nabla \theta_{k \varepsilon} \cdot \nabla \phi_{n}\left(\theta_{k \varepsilon}\right) \mathrm{d} x \mathrm{~d} t \\
& \leq \int_{Q} \mathbb{K} \nabla \theta_{k \varepsilon} \cdot \nabla \phi_{n}\left(\theta_{k \varepsilon}\right) \mathrm{d} x \mathrm{~d} t+\int_{\Omega} \widehat{\mathfrak{c} \phi_{n}}\left(\theta_{k \varepsilon}(T, \cdot)\right) \mathrm{d} x \\
& =\int_{\Omega} \widehat{\mathfrak{c} \phi_{n}}\left(\theta_{0, k}\right) \mathrm{d} x+\int_{Q} g_{k} \phi_{n}\left(\theta_{k \varepsilon}\right) \mathrm{d} x \mathrm{~d} t \\
& \quad+\int_{\Sigma} h_{k} \phi_{n}\left(\theta_{k \varepsilon}\right) \mathrm{d} S \mathrm{~d} t+\int_{Q} R_{k \varepsilon} \phi_{n}\left(\theta_{k \varepsilon}\right) \mathrm{d} x \mathrm{~d} t \\
& \leq c_{\max }\left(\left\|\theta_{0, k}\right\|_{L^{1}(\Omega)}+\frac{1}{\omega}\left\|\theta_{0, k}\right\|_{L^{\omega}(\Omega)}^{\omega}\right) \\
& \quad+\left\|g_{k}\right\|_{L^{1}(Q)}+\left\|h_{k}\right\|_{L^{1}(\Sigma)}+\left\|R_{k \varepsilon}\right\|_{L^{1}(Q)},
\end{aligned}
$$

where $\widehat{\mathfrak{c} \phi_{n}}$ is the primitive function of $\mathfrak{c} \phi_{n}$ such that $\widehat{\mathfrak{c}} \phi_{n}(0)=0$ and where $c_{\max }$ is from $(3.3 \mathrm{~h})$. Note that $\widehat{\boldsymbol{c}} \phi_{n}(\theta) \leq c_{\max }\left(\theta+\frac{1}{\omega} \theta^{\omega}\right)$ due to $(3.3 \mathrm{~h})$. For $\zeta>0$ fixed, we get

$$
\begin{aligned}
& \int_{Q} \frac{\left|\nabla \theta_{k \varepsilon}\right|^{2}}{\left(1+\theta_{k \varepsilon}\right)^{1+\zeta}} \mathrm{d} x \mathrm{~d} t=\sum_{n=0}^{\infty} \int_{B_{n}} \frac{\left|\nabla \theta_{k \varepsilon}\right|^{2}}{\left(1+\theta_{k \varepsilon}\right)^{1+\zeta}} \mathrm{d} x \mathrm{~d} t \\
& \leq \sum_{n=0}^{\infty} \frac{1}{(1+n)^{1+\zeta}} \int_{B_{n}}\left|\nabla \theta_{k \varepsilon}\right|^{2} \mathrm{~d} x \mathrm{~d} t \\
& \leq \frac{1}{\kappa} \sum_{n=0}^{\infty} \frac{1}{(1+n)^{1+\zeta}}\left(c_{\max }\left\|\theta_{0, k}\right\|_{L^{1}(\Omega)}+\frac{c_{\max }}{\omega}\left\|\theta_{0, k}\right\|_{L^{\omega}(\Omega)}^{\omega}\right. \\
& \left.\quad+\left\|g_{k}\right\|_{L^{1}(Q)}+\left\|h_{k}\right\|_{L^{1}(\Sigma)}+\left\|R_{k \varepsilon}\right\|_{L^{1}(Q)}\right) \\
& \quad=C_{13}+C_{14}\left\|R_{k \varepsilon}\right\|_{L^{1}(Q)}
\end{aligned}
$$

with $C_{13}$ and $C_{14}$ suitable constants:

$$
\begin{aligned}
C_{13} & :=C_{14} \sup _{k \in \mathbb{N}}\left(c_{\max }\left\|\theta_{0, k}\right\|_{L^{1}(\Omega)}+\frac{c_{\max }}{\omega}\left\|\theta_{0, k}\right\|_{L^{\omega}(\Omega)}^{\omega}+\left\|g_{k}\right\|_{L^{1}(Q)}+\left\|h_{k}\right\|_{L^{1}(\Sigma)}\right), \\
C_{14} & :=\frac{1}{\kappa} \sum_{n=0}^{\infty} \frac{1}{(1+n)^{1+\zeta}} .
\end{aligned}
$$

Take $1 \leq r<2$. By Hölder's inequality and (4.30),

$$
\begin{aligned}
& \int_{Q}\left|\nabla \theta_{k \varepsilon}\right|^{r} \mathrm{~d} x \mathrm{~d} t=\int_{Q} \frac{\left|\nabla \theta_{k \varepsilon}\right|^{r}}{\left(1+\theta_{k \varepsilon}\right)^{(1+\zeta) r / 2}}\left(1+\theta_{k \varepsilon}\right)^{(1+\zeta) r / 2} \mathrm{~d} x \mathrm{~d} t \\
& \quad \leq\left(\int_{Q} \frac{\left|\nabla \theta_{k \varepsilon}\right|^{2}}{\left(1+\theta_{k \varepsilon}\right)^{1+\zeta}} \mathrm{d} x \mathrm{~d} t\right)^{r / 2}\left(\int_{Q}\left(1+\theta_{k \varepsilon}\right)^{(1+\zeta) r /(2-r)} \mathrm{d} x \mathrm{~d} t\right)^{(2-r) / 2} \\
& \quad \leq\left(C_{13}+C_{14}\left\|R_{k \varepsilon}\right\|_{L^{1}(Q)}\right)^{r / 2}\left(\int_{0}^{T}\left\|1+\theta_{k \varepsilon}(t, \cdot)\right\|_{L^{(1+\zeta) r /(2-r)}(\Omega)}^{(1+\zeta) r /(2-r)} \mathrm{d} t\right)^{(2-r) / 2}
\end{aligned}
$$


Now, we use Gagliardo-Nirenberg's inequality with some $C_{\mathrm{GN}, 1} \in \mathbb{R}$ and the norm $\|\cdot\|_{L^{\omega}(\Omega)}+\|\nabla \cdot\|_{L^{r}\left(\Omega ; \mathbb{R}^{d}\right)}$ on $W^{1, r}(\Omega)$ :

$$
\begin{aligned}
&\left\|1+\theta_{k \varepsilon}(t, \cdot)\right\|_{L^{(1+\zeta) r /(2-r)}(\Omega)} \leq C_{\mathrm{GN}, 1}\left(\left\|1+\theta_{k \varepsilon}(t, \cdot)\right\|_{L^{\omega}(\Omega)}\right. \\
&\left.+\left\|\nabla \theta_{k \varepsilon}(t, \cdot)\right\|_{L^{r}\left(\Omega ; \mathbb{R}^{d}\right)}\right)^{\lambda}\left\|1+\theta_{k \varepsilon}(t, \cdot)\right\|_{L^{\omega}(\Omega)}^{1-\lambda} \\
& \leq C_{\mathrm{GN}, 1}\left(|\Omega|^{1 / \omega}+C_{8}\right)^{1-\lambda}\left(|\Omega|^{1 / \omega}+C_{8}+\left\|\nabla \theta_{k \varepsilon}(t, \cdot)\right\|_{L^{r}\left(\Omega ; \mathbb{R}^{d}\right)}\right)^{\lambda}
\end{aligned}
$$

for

$$
\frac{2-r}{(1+\zeta) r} \geq \lambda\left(\frac{1}{r}-\frac{1}{d}\right)+\frac{1-\lambda}{\omega}
$$

We raise (4.32) to the $(1+\zeta) r /(2-r)$ power, use it in (4.31), and choose $\lambda:=(2-r) /(1+\zeta)$ (note that $1 \leq r<2$ and $\zeta \geq 0$ imply $0<\lambda \leq 1$ ):

$$
\begin{aligned}
& \left(\int_{0}^{T}\left\|1+\theta_{k \varepsilon}(t, \cdot)\right\|_{L^{(1+\zeta) r /(2-r)}(\Omega)}^{(1+\zeta) r /(2-r)} \mathrm{d} t\right)^{(2-r) / 2} \\
& \leq\left(\int_{0}^{T} C_{\mathrm{GN}, 1}^{(1+\zeta) r /(2-r)}\left(|\Omega|^{1 / \omega}+C_{8}\right)^{(1-\lambda)(1+\zeta) r /(2-r)}\right. \\
& \left.\quad \times\left(|\Omega|^{1 / \omega}+C_{8}+\left\|\nabla \theta_{k \varepsilon}(t, \cdot)\right\|_{L^{r}\left(\Omega ; \mathbb{R}^{d}\right)}\right)^{\lambda(1+\zeta) r /(2-r)} \mathrm{d} t\right)^{(2-r) / 2} \\
& \leq\left(\int_{0}^{T} 3^{r-1} C_{\mathrm{GN}, 1}^{(1+\zeta) r /(2-r)}\left(|\Omega|^{1 / \omega}+C_{8}\right)^{(1-\lambda)(1+\zeta) r /(2-r)}\right. \\
& \left.\quad \times\left(|\Omega|^{r / \omega}+C_{8}^{r}+\left\|\nabla \theta_{k \varepsilon}(t, \cdot)\right\|_{L^{r}\left(\Omega ; \mathbb{R}^{d}\right)}^{r}\right) \mathrm{d} t\right)^{(2-r) / 2} \\
& =: C_{15}+C_{16}\left(\int_{Q}\left|\nabla \theta_{k \varepsilon}\right|^{r} \mathrm{~d} x \mathrm{~d} t\right)^{(2-r) / 2}
\end{aligned}
$$

for suitable constants $C_{15}$ and $C_{16}$. Joining (4.31) with (4.34) gives the estimate of the type

$$
\begin{aligned}
& \left\|\nabla \theta_{k \varepsilon}\right\|_{L^{r}\left(Q ; \mathbb{R}^{d}\right)}^{r} \leq C_{17} \\
& \quad+C_{18} \int_{Q}\left|\mathbb{D}\left(\frac{\partial e\left(u_{k \varepsilon}\right)}{\partial t}\right): \frac{\partial e\left(u_{k \varepsilon}\right)}{\partial t}-\theta_{k \varepsilon} \mathbb{C B}: \frac{\partial e\left(u_{k \varepsilon}\right)}{\partial t}\right| \mathrm{d} x \mathrm{~d} t
\end{aligned}
$$

with suitable constants $C_{17}$ and $C_{18}$. Substituting this choice of $\lambda:=(2-r) /(1+\zeta)$ into (4.33), one gets after some algebra the condition

$$
r \leq \frac{2 \omega+d-\zeta d}{\omega+d}
$$

Further, add (4.23) integrated over $[0, T]$ to (4.35) multiplied by a sufficiently small weight so that the dissipation term on the right-hand side $R_{k \varepsilon}$ in (4.30) can be dominated by the corresponding left-hand-side term in (4.23). Taking this weight as $1 /\left(2 C_{18}\right)$ thus 
gives

$$
\begin{aligned}
& \frac{\eta_{1}}{2}\left\|\frac{\partial e\left(u_{k \varepsilon}\right)}{\partial t}\right\|_{L^{p}\left(Q ; \mathbb{R}_{\mathrm{sym}}^{d \times d}\right)}^{p}+\frac{1}{2 C_{18}}\left\|\nabla \theta_{k \varepsilon}\right\|_{L^{r}\left(Q ; \mathbb{R}^{d}\right)}^{r} \\
& \quad \leq \int_{Q} \frac{1}{2} \mathbb{D}\left(\frac{\partial e\left(u_{k \varepsilon}\right)}{\partial t}\right): \frac{\partial e\left(u_{k \varepsilon}\right)}{\partial t}+\frac{1}{2 C_{18}}\left|\nabla \theta_{k \varepsilon}\right|^{r} \mathrm{~d} x \mathrm{~d} t \\
& \leq \frac{\rho}{2}\left\|v_{0, k}\right\|_{L^{2}\left(\Omega ; \mathbb{R}^{d}\right)}^{2}+\frac{1}{2} \int_{\Omega} \mathbb{C} e\left(u_{0, k}\right): e\left(u_{0, k}\right) \mathrm{d} x \\
&+\int_{Q} f_{k} \cdot \frac{\partial u_{k \varepsilon}}{\partial t} \mathrm{~d} x \mathrm{~d} t+\frac{C_{17}}{2 C_{18}}+\frac{3}{2}\left|\int_{Q} \theta_{k \varepsilon} \mathbb{C B}: \frac{\partial e\left(u_{k \varepsilon}\right)}{\partial t} \mathrm{~d} x \mathrm{~d} t\right|
\end{aligned}
$$

note that $\eta_{1}$ came from (3.3d) and that the term $\varepsilon\left|\frac{\partial}{\partial t} u_{k \varepsilon}\right|^{2 p}$ as well as the terms $\frac{\rho}{2}\left\|\frac{\partial}{\partial t} u_{k \varepsilon}(T, \cdot)\right\|_{L^{2}\left(\Omega ; \mathbb{R}^{d}\right)}^{2}$ and $\frac{1}{2} \int_{\Omega} \mathbb{C} e\left(u_{k \varepsilon}(T, \cdot)\right): e\left(u_{k \varepsilon}(T, \cdot)\right) \mathrm{d} x$ have been "forgotten". We estimate the last term in (4.37) by Hölder's and Young's inequalities as

$$
\frac{3}{2}\left|\int_{Q} \theta_{k \varepsilon} \mathbb{C B}: \frac{\partial e\left(u_{k \varepsilon}\right)}{\partial t} \mathrm{~d} x \mathrm{~d} t\right| \leq C_{\delta}\left\|\theta_{k \varepsilon}\right\|_{L^{p /(p-1)}(Q)}^{p /(p-1)}+\delta\left\|\frac{\partial e\left(u_{k \varepsilon}\right)}{\partial t}\right\|_{L^{p}\left(Q ; \mathbb{R}^{d \times d}\right)}^{p},
$$

where $C_{\delta}$ depends on $\mathbb{B}, \mathbb{C}$, and also on $\delta>0$. If $\delta>0$ is small, the last term can be absorbed in the left-hand side of (4.37). Further, using Gagliardo-Nirenberg's inequality once more yields:

$$
\begin{aligned}
& \left\|\theta_{k \varepsilon}(t, \cdot)\right\|_{L^{p /(p-1)}(\Omega)} \leq C_{\mathrm{GN}, 2}\left\|\theta_{k \varepsilon}(t, \cdot)\right\|_{L^{\omega}(\Omega)}^{1-\mu}\left(\left\|\theta_{k \varepsilon}(t, \cdot)\right\|_{L^{\omega}(\Omega)}\right. \\
& \left.\quad+\left\|\nabla \theta_{k \varepsilon}(t, \cdot)\right\|_{L^{r}\left(\Omega ; \mathbb{R}^{d}\right)}\right)^{\mu} \leq C_{\mathrm{GN}, 2} C_{8}^{1-\mu}\left(C_{8}+\left\|\nabla \theta_{k \varepsilon}(t, \cdot)\right\|_{L^{r}\left(\Omega ; \mathbb{R}^{d}\right)}\right)^{\mu}
\end{aligned}
$$

for

$$
\frac{p-1}{p} \geq \mu\left(\frac{1}{r}-\frac{1}{d}\right)+\frac{1-\mu}{\omega} .
$$

Now, raise (4.39) to the power $p /(p-1)$ and assume

$$
\frac{\mu p}{p-1}<r
$$

and integrate it over $[0, T]$ and use Young's inequality

$$
\begin{gathered}
\left\|\theta_{k \varepsilon}\right\|_{L^{p /(p-1)}(Q)}^{p /(p-1)} \leq C_{\mathrm{GN}, 2}^{p /(p-1)} C_{8}^{(1-\mu) p /(p-1)} \int_{0}^{T}\left(C_{8}+\left\|\nabla \theta_{k \varepsilon}(t, \cdot)\right\|_{L^{r}\left(\Omega ; \mathbb{R}^{d}\right)}\right)^{\mu p /(p-1)} \mathrm{d} t \\
\leq \int_{0}^{T}\left(C_{\delta}+\delta\left\|\nabla \theta_{k \varepsilon}(t, \cdot)\right\|_{L^{r}\left(\Omega ; \mathbb{R}^{d}\right)}^{r}\right) \mathrm{d} t=T C_{\delta}+\delta\left\|\nabla \theta_{k \varepsilon}\right\|_{L^{r}\left(Q ; \mathbb{R}^{d}\right)}^{r},
\end{gathered}
$$

where $C_{\delta}$ depends here, beside $\delta>0$, also on $C_{\mathrm{GN}, 2}, C_{8}, \mu, p$ and $r$. Substitute it into (4.38), and use it in (4.37). Choosing $\delta>0$ small, we can absorb both the already discussed right-hand-side term $\delta\left\|\frac{\partial}{\partial t} e\left(u_{k \varepsilon}\right)\right\|_{L^{p}\left(Q ; \mathbb{R}^{d \times d}\right)}^{p}$ of (4.38), and the right-hand-side term $\delta\left\|\nabla \theta_{k \varepsilon}\right\|_{L^{r}\left(\Omega ; \mathbb{R}^{d}\right)}^{r}$ in the left-hand side of (4.35). This eventually gives the estimates (4.21b and

$$
\left\|\nabla \theta_{k \varepsilon}\right\|_{L^{r}\left(Q ; \mathbb{R}^{d}\right)} \leq C_{17}
$$

Now, let us analyze the above conditions. From (4.41) with (4.40), one gets

$$
\frac{\mu}{r}>\frac{p-1}{p} \geq \frac{\mu}{r}-\frac{\mu}{d}+\frac{1-\mu}{\omega},
$$


which yields $\mu>d /(d+\omega)$. Substituting this condition, i.e. $\mu=d /(d+\omega)+\eta$ with $\eta>0$ and also (4.36) into (4.41) gives, after some algebra, eventually

$$
p>\frac{2 \omega+d-\zeta d}{2 \omega-\zeta d-\eta(\omega+d)} .
$$

Of course, we are allowed to take $\zeta>0$ and $\eta>0$ arbitrarily small, which eventually yields the condition (3.2).

The $\varepsilon$-terms in the right-hand-side terms in (4.26) give the estimate (4.22 a) and $\left\|\theta_{k \varepsilon}\right\|_{L^{2}(Q)}=\mathcal{O}\left(\varepsilon^{-1 / 2}\right)$. The latter estimate, though being uniform with respect to $k$ and $\varepsilon$, obviously just shows the term

$$
\varepsilon\left|\theta_{k \varepsilon}\right| \theta_{k \varepsilon} \quad \text { bounded in } L^{1}(\Omega)
$$

but this would not suffice for convergence of this term to zero for $\varepsilon \rightarrow 0$, as we will need in Lemma 4.4. For this, we need a "higher-power estimate", namely (4.22b), though dependent on $k$. We derive it by testing (4.17b) separately by $\theta_{k \varepsilon}$. It is important that we have $\left\|\frac{\partial}{\partial t} u_{k \varepsilon}\right\|_{L^{\infty}\left(I ; L^{2}\left(\Omega ; \mathbb{R}^{d}\right)\right)}$ bounded independently of $\varepsilon$, cf. (4.21a), and then, considering $k \in \mathbb{N}$ fixed so that the $L^{2}(\Omega)$ - and $W^{1, \infty}(\Omega)$-norms are mutually equivalent on the finite-dimensional space $V_{k}$, we have also $\left\|\frac{\partial}{\partial t} e\left(u_{k \varepsilon}\right)\right\|_{L^{\infty}\left(Q ; \mathbb{R}^{d \times d}\right)}$ bounded independently of $\varepsilon$ (though it may be dependent on $k$ ). Then (4.22 b) follows standardly.

The estimate (4.21 $\mathrm{c}$ ) follows from the already proved estimates $4.21 \mathrm{~b}, \mathrm{~b}, \mathrm{~d}, \mathrm{e})$. Also (4.21f) follows from (4.21 $\mathrm{b}, \mathrm{d}, \mathrm{e}$ ) and also from (4.46). This is a bit technical, however. As the right-hand side $R_{k \varepsilon}+g_{k}$ of (4.17b) belongs to $L^{2}(Q)$ and $h_{k} \in W^{1,2}\left(I ; L^{2}(\Gamma)\right)$ due to $(4.2 k)$, and as $-\operatorname{div}\left(\mathbb{K} \nabla \theta_{k \varepsilon}\right)+\left|\theta_{k \varepsilon}\right| \theta_{k \varepsilon}$ has a nonnegative potential $\Phi\left(\theta_{k \varepsilon}\right):=\int_{\Omega} \frac{1}{2} \mathbb{K} \nabla \theta_{k \varepsilon}$. $\nabla \theta_{k \varepsilon}+\frac{1}{3}\left|\theta_{k \varepsilon}\right|^{3} \mathrm{~d} x$ and $\Phi\left(\theta_{0, k}\right)<+\infty$ due to the assumed symmetry of $\mathbb{K}$ and due to $\theta_{0, k} \in V_{k} \subset W^{1, \infty}(\Omega)$ by (4.3k), we can test (4.17b) by $\frac{\partial}{\partial t} \theta_{k \varepsilon}$. This shows $\sqrt{\mathfrak{c}\left(\theta_{k \varepsilon}\right)} \frac{\partial}{\partial t} \theta_{k \varepsilon} \in$ $L^{2}(Q)$ and thus also $\frac{1}{\mathfrak{c}\left(\theta_{k \varepsilon}\right)} \operatorname{div}\left(\mathbb{K} \nabla \theta_{k \varepsilon}\right)=\frac{1}{\sqrt{\mathfrak{c}\left(\theta_{k \varepsilon}\right)}}\left(\sqrt{\mathfrak{c}\left(\theta_{k \varepsilon}\right)} \frac{\partial}{\partial t} \theta_{k \varepsilon}\right)-\frac{1}{\mathfrak{c}\left(\theta_{k \varepsilon}\right)}\left(R_{k \varepsilon}+g_{k}\right) \in$ $L^{2}(Q)$, although their norms in these spaces are not uniform with respect to $k$. Thus the following calculus applied to (4.17b) divided by $\mathfrak{c}\left(\theta_{k \varepsilon}\right)$ is legal:

$$
\begin{aligned}
\int_{Q} \frac{\partial \theta_{k \varepsilon}}{\partial t} & w \mathrm{~d} x \mathrm{~d} t=\int_{Q} \frac{R_{k \varepsilon}+g_{k}+\operatorname{div}\left(\mathbb{K} \nabla \theta_{k \varepsilon}\right)}{\mathfrak{c}\left(\theta_{k \varepsilon}\right)} w \mathrm{~d} x \mathrm{~d} t \\
\quad= & \int_{Q}\left(\frac{\left(R_{k \varepsilon}+g_{k}\right) w}{\mathfrak{c}\left(\theta_{k \varepsilon}\right)}-\mathbb{K} \nabla \theta_{k \varepsilon} \cdot \nabla \frac{w}{\mathfrak{c}\left(\theta_{k \varepsilon}\right)}\right) \mathrm{d} x \mathrm{~d} t \\
\quad= & \int_{Q}\left(\frac{\left(R_{k \varepsilon}+g_{k}\right) w}{\mathfrak{c}\left(\theta_{k \varepsilon}\right)}-\frac{\mathbb{K} \nabla \theta_{k \varepsilon} \cdot \nabla w}{\mathfrak{c}\left(\theta_{k \varepsilon}\right)}+\frac{\mathfrak{c}^{\prime}\left(\theta_{k \varepsilon}\right) \mathbb{K} \nabla \theta_{k \varepsilon} \cdot \nabla \theta_{k \varepsilon} w}{\mathfrak{c}\left(\theta_{k \varepsilon}\right)^{2}}\right) \mathrm{d} x \mathrm{~d} t
\end{aligned}
$$

for any $w \in L^{\infty}\left(I ; W_{0}^{1+d / 2,2}(\Omega)\right)$. Thus

$$
\begin{aligned}
& \left\|\frac{\partial \theta_{k \varepsilon}}{\partial t}\right\|_{L^{1}\left(I ; W^{-1-d / 2,2}(\Omega)\right)}=\sup _{w \in L^{\infty}\left(I ; W_{0}^{1+d / 2,2}(\Omega)\right)} \int_{Q} \frac{\partial \theta_{k \varepsilon}}{\partial t} w \mathrm{~d} x \mathrm{~d} t \\
& =\sup _{w \in L^{\infty}\left(I ; W_{0}^{1+d / 2,2}(\Omega)\right)} \int_{Q}\left(\frac{\left(R_{k \varepsilon}+g_{k}\right) w}{\mathfrak{c}\left(\theta_{k \varepsilon}\right)}-\frac{\mathbb{K} \nabla \theta_{k \varepsilon} \cdot \nabla w}{\mathfrak{c}\left(\theta_{k \varepsilon}\right)}+\frac{\mathfrak{c}^{\prime}\left(\theta_{k \varepsilon}\right) \mathbb{K} \nabla \theta_{k \varepsilon} \cdot \nabla \theta_{k \varepsilon} w}{\mathfrak{c}\left(\theta_{k \varepsilon}\right)^{2}}\right) \mathrm{d} x \mathrm{~d} t .
\end{aligned}
$$

Now we can estimate it by using $\nabla w$ bounded in $L^{\infty}\left(Q ; \mathbb{R}^{d}\right)$ and the already proved estimates (4.21 $\mathrm{h}, \mathrm{b}, \mathrm{d}, \mathrm{e})$ as well as by the assumption that $1 / \mathfrak{c}(\cdot)$ is bounded, cf. (3.3h). 
In particular, we estimate

$$
\begin{aligned}
\int_{Q} \frac{\mathfrak{c}^{\prime}\left(\theta_{k \varepsilon}\right) \mathbb{K} \nabla \theta_{k \varepsilon} \cdot \nabla \theta_{k \varepsilon} w}{\mathfrak{c}\left(\theta_{k \varepsilon}\right)^{2}} \mathrm{~d} x \mathrm{~d} t & \leq \int_{Q} C_{\max }|\mathbb{K}| \frac{\left|\nabla \theta_{k \varepsilon}\right|^{2}}{\left(1+\theta_{k \varepsilon}\right)^{1+\zeta}}|w| \mathrm{d} x \mathrm{~d} t \\
& \leq C_{\max }|\mathbb{K}|\left(C_{13}+C_{14}\left\|R_{k \varepsilon}\right\|_{L^{1}(Q)}\right)\|w\|_{L^{\infty}(Q)}
\end{aligned}
$$

with $C_{\max }$ from (3.3i) and $C_{13}$ and $C_{14}$ from (4.30), and the fact that $R_{k \varepsilon}$ is already proved bounded in $L^{1}(Q)$. Here we took $\zeta>0$ small enough to make (3.3i) effective. It is important that, though the regularity estimates that allowed for (4.47) were not uniform, the last estimate is again uniform with respect to both $\varepsilon$ and $k$.

Now, by passing $\varepsilon \rightarrow 0$, we want to get a solution, let us denote it by $\left(u_{k}, \theta_{k}\right)$, to a nonregularized semi-Galerkin problem, i.e. (4.17) but with $\varepsilon=0$ and thus the $\varepsilon$-terms as well as the indexes $\varepsilon$ omitted. It is important still to hold $k$ fixed because $4.22 \mathrm{~b}$ ) depends on $k$.

Lemma 4.4 (Passage $\varepsilon \rightarrow 0$ ). Let the assumptions of Proposition 4.3 hold. Then the sequence $\left\{\left(u_{k \varepsilon}, \theta_{k \varepsilon}\right)\right\}_{\varepsilon>0}$ contains a subsequence converging weakly* in the topologies induced by the estimates (4.18) to a limit $\left(u_{k}, \theta_{k}\right)$ which solves in the weak sense the semi-Galerkin nonregularized system, i.e.

$$
\begin{aligned}
& \varrho \frac{\partial^{2} u_{k}}{\partial t^{2}}-\operatorname{div}\left(\mathbb{C}\left(e\left(u_{k}\right)-\theta_{k} \mathbb{B}\right)+\mathbb{D}\left(\frac{\partial e\left(u_{k}\right)}{\partial t}\right)\right)=f_{k}+r_{k}, \\
& \mathfrak{c}\left(\theta_{k}\right) \frac{\partial \theta_{k}}{\partial t}-\operatorname{div}\left(\mathbb{K} \nabla \theta_{k \varepsilon}\right)=\mathbb{D}\left(\frac{\partial e\left(u_{k}\right)}{\partial t}\right): \frac{\partial e\left(u_{k}\right)}{\partial t}-\theta_{k} \mathbb{C B}: \frac{\partial e\left(u_{k}\right)}{\partial t}+g_{k},
\end{aligned}
$$

with the initial conditions $u_{k}(0, \cdot)=u_{0, k}, \frac{\partial u_{k}}{\partial t}(0, \cdot)=v_{0, k}$, and $\theta_{k}(0, \cdot)=\theta_{0, k}$ and the boundary conditions $\sigma_{k} \nu=0$ and $\mathbb{K} \frac{\partial}{\partial \nu} \theta_{k}=h_{k}$ on $\Sigma$ with $\sigma_{k}$ denoting the stress tensor as in (2.1 b) but with $u_{k}$ and $\theta_{k}$ in place of $u$ and $\theta$, respectively, and where the residuum $r_{k} \in W^{1, p}\left(\Omega ; \mathbb{R}^{d}\right)^{*}$ is orthogonal to $V_{k}^{d}$. Moreover, (4.21) is inherited for this limit and (4.21c) can be improved to

$$
\left|\frac{\partial^{2} u_{k}}{\partial t^{2}}\right|_{\widehat{k},\left(L^{p}\left(I ; W^{1, p}\left(\Omega ; \mathbb{R}^{d}\right)\right) \cap L^{\infty}\left(I ; L^{2}\left(\Omega ; \mathbb{R}^{d}\right)\right)\right)^{*}} \leq C_{18} \quad \text { for } \widehat{k} \geq k .
$$

Proof. Likewise in Lemma 4.2, this limit passage is still easy because $u_{k \varepsilon}$ ranges still a fixed finite-dimensional space $V_{k}^{d}$. The difference here is that the regularizing terms are now to be shown vanishing in the limit, which is due to the estimates

$$
\begin{array}{r}
\left.\left|\int_{Q} \varepsilon\right| \frac{\partial u_{k \varepsilon}}{\partial t}\right|^{2 p-2} \frac{\partial u_{k \varepsilon}}{\partial t} \cdot z \mathrm{~d} x \mathrm{~d} t\left|\leq \varepsilon\left\|\left|\frac{\partial u_{k \varepsilon}}{\partial t}\right|^{2 p-1}\right\|_{L^{2 p /(2 p-1)}(Q)}\|z\|_{L^{2 p}\left(Q ; \mathbb{R}^{d}\right)}\right. \\
=\varepsilon\left\|\frac{\partial u_{k \varepsilon}}{\partial t}\right\|_{L^{2 p}(Q)}^{2 p-1}\|z\|_{L^{2 p}\left(Q ; \mathbb{R}^{d}\right)}=\varepsilon^{1 /(2 p)} C_{11}^{2 p-1}\|z\|_{L^{2 p}\left(Q ; \mathbb{R}^{d}\right)}
\end{array}
$$

and

$$
\begin{aligned}
\left|\int_{Q} \varepsilon\right| \theta_{k \varepsilon}\left|\theta_{k \varepsilon} w \mathrm{~d} x\right| & \leq \varepsilon\left\|\theta_{k \varepsilon}^{2}\right\|_{L^{3 / 2}(Q)}\|w\|_{L^{3}(Q)} \\
& =\varepsilon\left\|\theta_{k \varepsilon}\right\|_{L^{3}(Q)}^{2}\|w\|_{L^{3}(Q)} \leq \varepsilon^{1 / 3} C_{12, k}^{2}\|w\|_{L^{3}(Q)},
\end{aligned}
$$


which both tend to 0 for $\varepsilon \rightarrow 0$ provided $k \in \mathbb{N}$ and $z \in C\left(\bar{Q} ; \mathbb{R}^{d}\right)$ or $w \in C(\bar{Q})$ are fixed; here $C_{11}$ and $C_{12, k}$ are from $(4.22 \mathrm{a}$ ) and $4.22 \mathrm{~b}$ ), respectively. We thus get a weak solution $\left(u_{k}, \theta_{k}\right)$ to the nonregularized semi-Galerkin problem (4.49).

The equation (4.49a) now allows for a test by functions from $L^{p}\left(I ; W^{1, p}\left(\Omega ; \mathbb{R}^{d}\right)\right) \cap$ $L^{\infty}\left(I ; L^{2}\left(\Omega ; \mathbb{R}^{d}\right)\right)$ valued in $V_{k}^{d}$ which gives (4.50).

REMARK 4.5. The equation (4.26) represents an energy balance (cf. also (4.57) below) where the $\varepsilon$-term represents an artificial dissipated energy related with our regularization. It is interesting that this artificial dissipated energy converges to zero. Indeed,

$$
\begin{aligned}
& \limsup _{\varepsilon \rightarrow 0} \varepsilon \int_{Q}\left(\left|\frac{\partial u_{k \varepsilon}}{\partial t}\right|^{2 p}+\left|\theta_{k \varepsilon}\right| \theta_{k \varepsilon}\right) \mathrm{d} x \mathrm{~d} t \\
&=-\liminf _{\varepsilon \rightarrow 0} \int_{\Omega}\left(\frac{\rho}{2}\left|\frac{\partial u_{k \varepsilon}}{\partial t}(T)\right|^{2}+\frac{1}{2} \mathbb{C} e\left(u_{k \varepsilon}(T)\right): e\left(u_{k \varepsilon}(T)\right)+\widehat{\mathfrak{c}}\left(\theta_{k \varepsilon}(T)\right)\right) \mathrm{d} x \\
& \quad+\int_{\Omega}\left(\frac{\rho}{2}\left|v_{0, k}\right|^{2}+\frac{1}{2} \mathbb{C} e\left(u_{0, k}\right): e\left(u_{0, k}\right)+\widehat{\mathfrak{c}}\left(\theta_{0, k}\right)\right) \mathrm{d} x \\
& \quad+\int_{Q}\left(f_{k} \cdot \frac{\partial u_{k \varepsilon}}{\partial t}+g_{k}\right) \mathrm{d} x \mathrm{~d} t+\int_{\Sigma} h_{k} \mathrm{~d} S \mathrm{~d} t \\
& \leq- \int_{\Omega}\left(\frac{\rho}{2}\left|\frac{\partial u_{k}}{\partial t}(T)\right|^{2}+\frac{1}{2} \mathbb{C} e\left(u_{k}(T)\right): e\left(u_{k}(T)\right)+\widehat{\mathfrak{c}}\left(\theta_{k}(T)\right)+\frac{\rho}{2}\left|v_{0, k}\right|^{2}\right. \\
&\left.+\frac{1}{2} \mathbb{C} e\left(u_{0, k}\right): e\left(u_{0, k}\right)+\widehat{\mathfrak{c}}\left(\theta_{0, k}\right)\right) \mathrm{d} x+\int_{Q}\left(f_{k} \cdot \frac{\partial u_{k}}{\partial t}+g_{k}\right) \mathrm{d} x \mathrm{~d} t+\int_{\Sigma} h_{k} \mathrm{~d} S \mathrm{~d} t=0
\end{aligned}
$$

where the last equation can be obtained by summing (4.49a) tested by $\frac{\partial u_{k}}{\partial t}$ and (4.49b) tested by 1. For this equality, it is important that $\frac{\partial^{2} u_{k}}{\partial t^{2}}$ is in duality with $\frac{\partial u_{k}}{\partial t} \in$ $L^{p}\left(I ; W^{1, p}\left(\Omega ; \mathbb{R}^{d}\right)\right)$ because of (4.50) and (4.21 a,b) inherited for $u_{k}$. Similarly, $\frac{\partial \theta_{k}}{\partial t}$ is in duality with 1 due to (4.21f) inherited for $\theta_{k}$.

For the limit passage in the approximate solutions, the only essential point is now to show the strong convergence of $\frac{\partial e(u)}{\partial t}$ in $L^{p}\left(Q ; \mathbb{R}^{d \times d}\right)$. For this, $d$-monotonicity assumed in (3.3f) is to be used.

Proposition 4.6 (Passage $k \rightarrow \infty$, existence of a solution). Let the assumptions of Proposition 4.3 hold. Then the sequence $\left\{\left(u_{k}, \theta_{k}\right)\right\}_{k \in \mathbb{N}}$ contains a subsequence converging weakly* in the topologies induced by the estimates (4.18) and (4.50) to a limit $(u, \theta)$ which solves the original boundary-value problem in the sense of Definition 3.1 and also (3.9) holds.

Proof. Let us abbreviate $v_{k}:=\frac{\partial}{\partial t} u_{k}$ and $v:=\frac{\partial}{\partial t} u$. As in the proof of Lemma 4.2, the uniform estimate (4.50) allows for successive extensions of $\frac{\partial^{2}}{\partial t^{2}} u=\frac{\partial}{\partial t} v$ to obtain $\frac{\partial}{\partial t} v \in\left(L^{p}\left(I ; W^{1, p}\left(\Omega ; \mathbb{R}^{d}\right)\right) \cap L^{\infty}\left(I ; L^{2}\left(\Omega ; \mathbb{R}^{d}\right)\right)\right)^{*}$. 
For the limit passage in the heat equation, we must ultimately have the strong convergence of $\frac{\partial}{\partial t} e\left(u_{k}\right)$ in $L^{p}\left(Q ; \mathbb{R}_{\mathrm{sym}}^{d \times d}\right)$. We use integration by parts:

$$
\begin{aligned}
& \int_{0}^{T}\left\langle\frac{\partial\left(v_{k}-v\right)}{\partial t}, v_{k}-v\right\rangle \mathrm{d} t \\
& =\int_{0}^{T}\left(\left\langle\frac{\partial v_{k}}{\partial t}, v_{k}\right\rangle+\left\langle\frac{\partial v}{\partial t}, v\right\rangle-\left\langle\frac{\partial v}{\partial t}, v_{k}\right\rangle-\left\langle\frac{\partial v_{k}}{\partial t}, v\right\rangle\right) \mathrm{d} t \\
& =\frac{1}{2}\left\|v_{k}(T, \cdot)\right\|_{L^{2}(\Omega)}^{2}-\frac{1}{2}\left\|v_{0, k}\right\|_{L^{2}(\Omega)}^{2}+\frac{1}{2}\|v(T, \cdot)\|_{L^{2}(\Omega)}^{2}-\frac{1}{2}\left\|v_{0}\right\|_{L^{2}(\Omega)}^{2} \\
& \quad-\int_{\Omega}\left(v_{k}(T, \cdot) \cdot v(T, \cdot)-v_{0, k} \cdot v_{0}\right) \mathrm{d} x \\
& =\frac{1}{2}\left\|v_{k}(T, \cdot)-v(T, \cdot)\right\|_{L^{2}(\Omega)}^{2}-\frac{1}{2}\left\|v_{0, k}-v_{0}\right\|_{L^{2}(\Omega)}^{2} \geq-\frac{1}{2}\left\|v_{0, k}-v_{0}\right\|_{L^{2}(\Omega)}^{2} \cdot
\end{aligned}
$$

Here it was important that $\frac{\partial}{\partial t} v$ is in duality to $v \in L^{p}\left(I ; W^{1, p}\left(\Omega ; \mathbb{R}^{d}\right)\right)$. Furthermore, the meaning of $\frac{\partial v_{k}}{\partial t}$ in the expression $\int_{0}^{T}\left\langle\frac{\partial v_{k}}{\partial t}, v\right\rangle \mathrm{d} t$ contained in (4.51) is a HahnBanach extension of $\frac{\partial v_{k}}{\partial t}$, being a bounded linear functional on $L^{p}\left(I ; V_{k}^{d}\right)$, onto all of $L^{p}\left(I ; W^{1, p}\left(\Omega ; \mathbb{R}^{d}\right)\right)$ but, anyhow, the value of $\int_{0}^{T}\left\langle\frac{\partial v_{k}}{\partial t}, v\right\rangle \mathrm{d} t$ is independent of the particular extension and in (4.51) we used that, in fact, it just equals

$$
\int_{0}^{T}\left\langle\frac{\partial v_{k}}{\partial t}, v\right\rangle \mathrm{d} t=\int_{\Omega}\left(v_{k}(T, \cdot) \cdot v(T, \cdot)-v_{0, k} \cdot v_{0}\right) \mathrm{d} x-\int_{0}^{T}\left\langle\frac{\partial v}{\partial t}, v_{k}\right\rangle \mathrm{d} t
$$

Linearity of the elastic response allows for

$$
\begin{aligned}
\int_{Q} \mathbb{C} e\left(u_{k}-u\right): e\left(v_{k}-v\right) \mathrm{d} x \mathrm{~d} t & =\frac{1}{2} \int_{0}^{T} \frac{\mathrm{d}}{\mathrm{d} t}\left(\int_{\Omega} \mathbb{C} e\left(u_{k}-u\right): e\left(u_{k}-u\right) \mathrm{d} x\right) \mathrm{d} t \\
& \geq-\frac{1}{2} \int_{\Omega} \mathbb{C} e\left(u_{0, k}-u_{0}\right):\left(u_{0, k}-u_{0}\right) \mathrm{d} x \\
& \geq-\frac{1}{2}|\mathbb{C}|\left\|e\left(u_{0, k}-u_{0}\right)\right\|_{L^{2}\left(\Omega ; \mathbb{R}_{\text {sym }}^{d \times d}\right)}^{2} .
\end{aligned}
$$

We take some approximation $w_{k}$ in $L^{\infty}\left(I ; W^{1, p}\left(\Omega ; \mathbb{R}^{d}\right)\right)$ of $v$ valued in $V_{k}^{d}$ such that $w_{k} \rightarrow v$ strongly in $L^{p}\left(I ; W^{1, p}\left(\Omega ; \mathbb{R}^{d}\right)\right) \cap L^{\infty}\left(I ; L^{2}\left(\Omega ; \mathbb{R}^{d}\right)\right)$. The existence of such an approximation can be seen by the density of $L^{\infty}\left(I ; W^{1, p}\left(\Omega ; \mathbb{R}^{d}\right)\right)$ in $L^{p}\left(I ; W^{1, p}\left(\Omega ; \mathbb{R}^{d}\right)\right)$, by the possibility of approximation of functions from $L^{\infty}\left(I ; W^{1, p}\left(\Omega ; \mathbb{R}^{d}\right)\right)$ by simple functions valued in $W^{1, p}\left(\Omega ; \mathbb{R}^{d}\right)$, and eventually by the possibility of approximation in the norm of $L^{\infty}\left(I ; W^{1, p}\left(\Omega ; \mathbb{R}^{d}\right)\right)$ of such functions by functions valued in $V_{k}^{d}$ because of (4.1). Having such a sequence $\left\{w_{k}\right\}_{k \in \mathbb{N}}$, by using also the Galerkin identity for $u_{k}$, i.e.

$$
\int_{0}^{T}\left(\varrho\left\langle\frac{\partial v_{k}}{\partial t}, z\right\rangle+\int_{\Omega}\left(\mathbb{D}\left(e\left(v_{k}\right)\right)+\mathbb{C}\left(e\left(u_{k}\right)\right)\right): e(z) \mathrm{d} x\right) \mathrm{d} t=\int_{Q}\left(f_{k} \cdot z+\theta_{k} \mathbb{C B B}: e(z)\right) \mathrm{d} x \mathrm{~d} t
$$


for any $z \in L^{p}\left(I ; V_{k}^{d}\right)$, in particular for $z:=v_{k}-w_{k}$, and by using the uniform monotonicity (3.3f), we obtain

$$
\begin{aligned}
\eta_{2} \| & \left(v_{k}-v\right) \|_{L^{p}\left(Q ; \mathbb{R}_{\text {sym }}^{d \times d}\right)}^{p} \leq \int_{Q}\left(\mathbb{D}\left(e\left(v_{k}\right)\right)-\mathbb{D}(e(v))\right): e\left(v_{k}-v\right) \mathrm{d} x \mathrm{~d} t \\
& \leq \int_{0}^{T}\left(\varrho\left\langle\frac{\partial v_{k}}{\partial t}-\frac{\partial v}{\partial t}, v_{k}-v\right\rangle\right. \\
& \left.+\int_{\Omega}\left(\left(\mathbb{D}\left(e\left(v_{k}\right)\right)-\mathbb{D}(e(v))\right)+\mathbb{C} e\left(u_{k}-u\right)\right): e\left(v_{k}-v\right) \mathrm{d} x\right) \mathrm{d} t \\
& +\frac{\varrho}{2}\left\|v_{0, k}-v_{0}\right\|_{L^{2}(\Omega)}^{2}+\frac{1}{2}|\mathbb{C}|\left\|e\left(u_{0, k}-u_{0}\right)\right\|_{L^{2}\left(\Omega ; \mathbb{R}_{\text {sym }}^{d \times d}\right)}^{2} \\
& =\int_{Q} \theta_{k} \mathbb{C B}: e\left(w_{k}-v_{k}\right) \mathrm{d} x \mathrm{~d} t+\int_{Q} f_{k} \cdot\left(w_{k}-v_{k}\right) \mathrm{d} x \mathrm{~d} t-\int_{0}^{T} \varrho\left\langle\frac{\partial v}{\partial t}, v_{k}-v\right\rangle \mathrm{d} t \\
& +\int_{Q}(\mathbb{D}(e(v))+\mathbb{C} e(u)): e\left(v_{k}-v\right) \mathrm{d} x \mathrm{~d} t+\int_{0}^{T} \varrho\left\langle\frac{\partial v_{k}}{\partial t}, w_{k}-v\right\rangle \mathrm{d} t \\
& +\int_{Q}\left(\mathbb{D}\left(e\left(v_{k}\right)\right)+\mathbb{C} e\left(u_{k}\right)\right): e\left(w_{k}-v\right) \mathrm{d} x \mathrm{~d} t=: \sum_{\ell=1}^{6} I_{k}^{(\ell)} .
\end{aligned}
$$

We push all the integrals $I_{k}^{(\ell)}$ to zero.

First, we can modify (4.31)-4.34) to get an "anisotropic" estimate

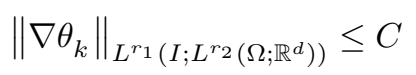

provided

$$
\frac{2}{r_{1}}+\frac{d}{r_{2}}>d+1, \quad r_{1}, r_{2} \geq 1
$$

we refer to [3, Formula (1.8)] and the proof therein. Then $I_{k}^{(1)} \rightarrow 0$ because $\theta_{k} \rightarrow \theta$ in $L^{p /(p-1)}(Q)$ provided (3.2) while $e\left(v_{k}-w_{k}\right) \rightarrow e(v-v)=0$ weakly in $L^{p}\left(Q ; \mathbb{R}_{\mathrm{sym}}^{d \times d}\right)$. The proof used strong convergence $\theta_{k} \rightarrow \theta$ followed by Aubin-Lions' theorem from the estimate of $\nabla \theta_{k}$ in $L^{p /(p-1)}\left(I ; W^{r_{2}}\left(\Omega ; \mathbb{R}^{d}\right)\right)$ with $r_{2}<p d /(p d-p+2)$, cf. (4.55)-44.56) with $r_{1}:=p /(p-1)$, so that $\theta_{k}$ is bounded in $L^{p /(p-1)}\left(I ; L_{2}^{r_{2}^{*}}(\Omega)\right)$ with $r_{2}^{*}$ the Sobolev exponent related to $r_{2}$, i.e. $r_{2}^{*}<d /(d-2)$ ( or $r_{2}^{*}=+\infty$ if $d=1$ ), and after some algebra one can see that $L^{r_{2}^{*}}(\Omega)$ is compactly embedded into $L^{p /(p-1)}(\Omega)$ just if (3.2) holds.

Furthermore, it follows that $I_{k}^{(2)} \rightarrow 0$ because of (4.2 a) and $w_{k}-v_{k} \rightarrow 0$ weakly* in $L^{\infty}\left(I ; L^{2}\left(\Omega ; \mathbb{R}^{d}\right)\right)$. Also, both $I_{k}^{(3)} \rightarrow 0$ and $I_{k}^{(4)} \rightarrow 0$ because $v_{k} \rightarrow v$ weakly in $L^{p}\left(I ; W^{1, p}\left(\Omega ; \mathbb{R}^{d}\right)\right)$ and $\frac{\partial v}{\partial t} \in L^{p}\left(I ; W^{1, p}\left(\Omega ; \mathbb{R}^{d}\right)\right)^{*}$ and $\mathbb{D}(e(v)) \in L^{p /(p-1)}\left(Q ; \mathbb{R}_{\mathrm{sym}}^{d \times d}\right)$ is in duality with $e\left(v_{k}-v\right) \in L^{p}\left(Q ; \mathbb{R}_{\mathrm{sym}}^{d \times d}\right)$. Note that $\mathbb{C} e(u) \in L^{\infty}\left(I ; L^{2}\left(\Omega ; \mathbb{R}_{\mathrm{sym}}^{d \times d}\right)\right)$ is indeed in duality with $e\left(v_{k}-v\right) \in L^{p}\left(I ; L^{p}\left(\Omega ; \mathbb{R}_{\mathrm{sym}}^{d \times d}\right)\right)$ because $p \geq 2$ is assumed in (3.2). As for $I_{k}^{(5)}$, we estimate

$$
\left|\int_{0}^{T} \varrho\left\langle\frac{\partial v_{k}}{\partial t}, w_{k}-v\right\rangle \mathrm{d} t\right| \leq\left\|\frac{\partial v_{k}}{\partial t}\right\|_{L^{p}\left(I ; W^{1, p}\left(\Omega ; \mathbb{R}^{d}\right)\right)^{*}}\left\|w_{k}-v\right\|_{L^{p}\left(I ; W^{1, p}\left(\Omega ; \mathbb{R}^{d}\right)\right)} \rightarrow 0,
$$

where $\frac{\partial v_{k}}{\partial t}$ means, like before, the norm-preserving Hahn-Banach extension of $\frac{\partial v_{k}}{\partial t} \in$ $L^{p}\left(I ; V_{k}^{d}\right)^{*}$ onto $L^{p}\left(I ; W^{1, p}\left(\Omega ; \mathbb{R}^{d}\right)\right)^{*}$. Eventually, $I_{k}^{(6)} \rightarrow 0$ because $e\left(w_{k}-v\right) \rightarrow 0$ in 
$L^{p}\left(Q ; \mathbb{R}_{\mathrm{sym}}^{d \times d}\right) \subset L^{2}\left(Q ; \mathbb{R}_{\mathrm{sym}}^{d \times d}\right)$ and $\mathbb{D}\left(e\left(v_{k}\right)\right)+\mathbb{C} e\left(u_{k}\right)$ is bounded in $L^{2}\left(Q ; \mathbb{R}_{\mathrm{sym}}^{d \times d}\right) ;$ here again $p \geq 2$ is used.

By having shown the convergence of the right-hand side of (4.54) to zero, we can conclude the strong convergence $e\left(v_{k}\right) \rightarrow e(v)$ in $L^{p}\left(Q ; \mathbb{R}_{\mathrm{sym}}^{d \times d}\right)$.

Now we test (4.49a) by $z_{l} \in C^{\infty}\left(I ; V_{l}^{d}\right)$ with $z_{l}(T, \cdot)=0$ and $l \in \mathbb{N}$ fixed and 4.49b) by $w \in C^{\infty}(Q)$ with $w(T, \cdot)=0$. Due to the estimates (4.50) as well as (4.21f), we can perform the integration by parts in time; in fact, (4.18d) allows for this step already for $\theta_{k \varepsilon}$. Having also the strong convergences of $\theta_{k}$, the limit passage to (3.7)-(3.8) is then simple. Eventually, we can pass $v_{l}$ to an arbitrary $z \in C^{\infty}\left(Q ; \mathbb{R}^{d}\right)$ with $z(T, \cdot)=0$ as used in (3.7).

The information $(3.9 \mathrm{a}, \mathrm{b})$ is obtained from the estimates $4.21 \mathrm{f})$ and $4.50 \mathrm{l})$. The fact that $\frac{\partial^{2}}{\partial t^{2}} u$ does not contract but remains in $L^{p /(p-1)}\left(I ; W^{1, p}\left(\Omega ; \mathbb{R}^{d}\right)^{*}\right) \cap L^{1}\left(I ; L^{2}\left(\Omega ; \mathbb{R}^{d}\right)\right)$ in $(3.9 \mathrm{a})$ follows from the fact that $\frac{\partial^{2}}{\partial t^{2}} u=(f+\operatorname{div} \sigma) / \varrho$ in the sense of distributions, see (2.1), and that $f \in L^{1}\left(I ; L^{2}\left(\Omega ; \mathbb{R}^{d}\right)\right)$ and $\sigma \in L^{p /(p-1)}\left(Q ; \mathbb{R}^{d \times d}\right)$; note that also $\theta \in L^{p /(p-1)}(Q)$ as argued in the proof of Proposition 4.6.

REMARK 4.7 (Energy balance (4.26)). The limit solutions satisfy the energy balance

$$
\begin{aligned}
& \frac{\mathrm{d}}{\mathrm{d} t} \int_{\Omega} \underbrace{\frac{\rho}{2}\left|\frac{\partial u}{\partial t}\right|^{2}}_{\begin{array}{c}
\text { specific } \\
\text { kinetic energy }
\end{array}}+\underbrace{\frac{1}{2} \mathbb{C} e(u): e(u)}_{\begin{array}{c}
\text { specific } \\
\text { stored energy }
\end{array}}+\underbrace{\widehat{\mathfrak{c}}(\theta)}_{\begin{array}{c}
\text { specific inter- } \\
\text { nal energy }
\end{array}} \mathrm{d} x \\
& =\underbrace{\int_{\Omega} f \cdot \frac{\partial u}{\partial t} \mathrm{~d} x}_{\begin{array}{c}
\text { power of exter- } \\
\text { nal force }
\end{array}}+\underbrace{\int_{\Omega} g \mathrm{~d} x+\int_{\Gamma} h \mathrm{~d} S}_{\begin{array}{c}
\text { power of exter- } \\
\text { nal heating }
\end{array}},
\end{aligned}
$$

which can be shown by summing the mechanical equation (3.7) tested by $z=\frac{\partial u}{\partial t}$ and the heat equation (3.8) tested by $w=1$. For this equality, it is important that $\frac{\partial^{2} u_{k}}{\partial t^{2}}$ is in duality with $\frac{\partial u_{k}}{\partial t} \in L^{p}\left(I ; W^{1, p}\left(\Omega ; \mathbb{R}^{d}\right)\right) \cap L^{\infty}\left(I ; L^{2}\left(\Omega ; \mathbb{R}^{d}\right)\right)$ because of $(3.9 \mathrm{k})$ so that we can extend (3.7) for such a test functions and perform the integration by parts in time. Similarly, $\frac{\partial}{\partial t} \widehat{\mathfrak{c}}(\theta)$ is in duality with 1 due to $(\underline{3.9}$ r).

REMARK 4.8 (The restriction (3.2) on $p$ and $\omega$ ). One might be tempted to use an anisotropic estimate (4.55) of $\nabla \theta$. Note that the condition (4.56) coincides with (4.36) in the special case $r=r_{1}=r_{2}$. It is, however, a sad reality that this technique does not improve (3.2). Hence, after an interpolation with the $L^{\infty}\left(I ; L^{\omega}(\Omega)\right)$-information as in (4.39), the isotropic estimate of $\theta$ in $L^{p /(p-1)}(Q)$ for $p>1+d /(2 \omega)$, cf. (3.2) and (4.42), is not improvable, which then also determines that $\frac{\partial}{\partial t} e(u)$ must be in $L^{p}\left(Q ; \mathbb{R}_{\mathrm{sym}}^{d \times d}\right)$ to give a sense to the adiabatic term $\theta \mathbb{C B B}: \frac{\partial}{\partial t} e(u)$ in $L^{1}(Q)$. Thus, the bound (3.2) seems to be optimal, at least if the $L^{1}$-data $g, h$, and $u_{0}$ are considered.

REMARK 4.9 (Nonlinear elastic response). A natural question arises why the elastic response through $\mathbb{C}$ is linear while the dissipation through $\mathbb{D}$ is not. Considering the stored energy nonquadratic leads to a much more complicated theory even in the isothermal case and requires also $\mathbb{D}$ to have a (pseudo)potential, as is well documented in [12], and its extension to the anisothermal case is not obvious. 
REMARK 4.10 (Nonlocal linear viscosity). We saw that the condition (3.2) allows for linear viscosity (i.e. $p=2$ ) only in the one-dimensional case. There is another option for introducing more viscosity into the system than implemented above, i.e. faster processes dissipate more due to the super-linear growth of $\mathbb{D}(\cdot)$ if $p>2$, namely that processes whose velocity gradient varies faster in space dissipate more. This might be justified by similar arguments for stored energy [25, 27] and a certain principle that any stored mechanism in nature must be at least a bit dissipative (perhaps with only a few exceptions of quantum origin like superconductivity). Such a nonlocal mechanism would give an additional contribution to the stress $\sigma$ in (2.1) of the form

$$
\left[\sigma_{\text {nonlocal }}(\dot{e})\right](x):=\int_{\Omega} K(x, \tilde{x})(\dot{e}(x)-\dot{e}(\tilde{x})) \mathrm{d} \tilde{x} \quad \text { with } \dot{e}=\frac{\partial e(u)}{\partial t} .
$$

The assumption on the kernel $K$ is

$$
\exists \zeta_{1}, \zeta_{2}>0: \quad \zeta_{1} \frac{\delta_{i k} \delta_{j l}}{|x-\tilde{x}|^{d+2 \gamma}} \leq K_{i j k l}(x, \tilde{x}) \leq \zeta_{2} \frac{\delta_{i k} \delta_{j l}}{|x-\tilde{x}|^{d+2 \gamma}}
$$

for a fixed parameter $0<\gamma<1$ where the $\delta$ 's denote here the Kronecker symbol. Then $\int_{\Omega} \sigma_{\text {nonlocal }}(\dot{e}): \dot{e} \mathrm{~d} x$ is equivalent to the square of the seminorm in the SobolevSlobodeckil spaces $W^{\gamma, 2}\left(\Omega ; \mathbb{R}_{\mathrm{sym}}^{d \times d}\right)$. Such a contribution would thus be majorized by the left-hand side of (correspondingly modified) (4.37) and the last term in (4.38) has still to be interpolated. We can do this in two steps. First, we use the interpolation between Hilbert-type Sobolev-Slobodetskil spaces applied on the symmetric part $e\left(\frac{\partial u}{\partial t}\right)$ of $\nabla\left(\frac{\partial u}{\partial t}\right)$, namely

$$
\left\|\frac{\partial e(u)}{\partial t}\right\|_{W^{\beta, 2}\left(\Omega ; \mathbb{R}_{\mathrm{sym}}^{d \times d}\right)} \leq C_{\mathrm{GN}, 3}\left\|\frac{\partial u}{\partial t}\right\|_{L^{2}\left(\Omega ; \mathbb{R}^{d}\right)}^{1-\nu}\left\|\frac{\partial e(u)}{\partial t}\right\|_{W^{\gamma, 2}\left(\Omega ; \mathbb{R}_{\mathrm{sym}}^{d \times d}\right)}^{\nu}
$$

with $0 \leq \beta=(1+\gamma) \nu-1$. Second, we use the continuous-embedding theorem for the Sobolev-Slobodetskiu spaces $W^{\beta, 2}(\Omega) \subset L^{p}(\Omega)$ provided $p \leq 2 d /(d-2 \beta)$. Joining these estimates, we obtain a Gagliardo-Nirenberg inequality generalized for fractional derivatives

$$
\left\|\frac{\partial e(u)}{\partial t}\right\|_{L^{p}\left(\Omega ; \mathbb{R}_{\mathrm{sym}}^{d \times d}\right)} \leq C_{\mathrm{GN}, 3}\left\|\frac{\partial u}{\partial t}\right\|_{L^{2}\left(\Omega ; \mathbb{R}^{d}\right)}^{1-\nu}\left\|\frac{\partial e(u)}{\partial t}\right\|_{W^{\gamma, 2}\left(\Omega ; \mathbb{R}_{\mathrm{sym}}^{d \times d}\right)}^{\nu}
$$

for

$$
\frac{1}{p} \geq \frac{1}{d}-\nu \frac{1+\gamma}{d}+\frac{1}{2}, \quad \frac{1}{1+\gamma} \leq \nu \leq 1
$$

To use it for further estimation of (4.38), we raise it to the power $p$ and then we need $\nu p<2$ to apply Young's inequality and absorb the thus arising term $\left\|\frac{\partial}{\partial t} e(u)\right\|_{W^{\gamma, 2}\left(\Omega ; \mathbb{R}_{\text {sym }}^{d \times d}\right)}^{2}$ in the correspondingly modified left-hand side of (4.37). Taking into account (3.2), we get $\nu<4 \omega /(d+2 \omega)$, and then (4.62) yields the restriction $\gamma>\left(d^{2}+2 d-4 \omega-2 \omega d\right) /(8 \omega)$ if $d \geq 2$. For the physically relevant case $d=3$, we have $\gamma>5 / 8$ if $\omega=1$ or $\gamma>0$ if $\omega=3 / 2$, while for the two-dimensional case we get $\gamma>0$ already for $\omega=1$.

REMARK 4.11 (Temperature dependence of the coefficients). The method presented above allows relatively easily for a generalization to a "more coupled" system using temperature-dependent $\mathbb{B}$ and $\mathbb{D}$ assuming that this dependence would be continuous, $\mathbb{B}(\cdot)$ bounded, and (3.3 $\mathrm{d}-\mathrm{f})$ would hold uniformly with respect to the temperature. The 
temperature dependence of $\mathbb{D}$ would require a slight enhancement of (4.54). On the other hand, generalization for temperature dependence of $\mathbb{K}$ is not clear though here it would be in conflict only with deriving (4.47) by using estimates obtained by the test by $\frac{\partial \theta}{\partial t}$.

\section{REFERENCES}

[1] J.-P. Aubin, Un théorème de compacité. C.R. Acad. Sci. 256 (1963), 5042-5044. MR0152860 $(27: 2832)$

[2] D. Blanchard, O. Guibé, Existence of a solution for a nonlinear system in thermoviscoelasticity. Adv. Diff. Eq. 5 (2000), 1221-1252. MR1785674 (2001h:74030)

[3] L. Boccardo, A. Dall'aglio, T. Gallouët, L. Orsina, Nonlinear parabolic equations with measure data. J. Funct. Anal. 147 (1997), 237-258. MR1453181(98c:35093)

[4] L. Boccardo, T. Gallouët, Non-linear elliptic and parabolic equations involving measure data. $J$. Funct. Anal. 87 (1989), 149-169. MR.1025884(92d:35286)

[5] M. Brokate, J. Sprekels, Hysteresis and Phase Transitions. Springer, New York, 1996. MR1411908 (97g:35127)

[6] Z. Chen, K.-H. Hoffmann: On a one-dimensional nonlinear thermoviscoelastic model for structural phase transitions in shape memory alloys. J. Diff. Equations 12 (1994), 325-350. MR1293474 (95f:35254)

[7] Z. Chen, K.-H. Hoffmann: Asymptotic behaviors of Landau-Devonshire-Ginzburg model for structural phase transitions in shape memory alloys. Adv. Math. Sci. Appl. 4 (1994), 209-226. MR.1287915 (95e:35210)

[8] C.M. Dafermos, Global smooth solutions to the initial boundary value problem for the equations of one-dimensional thermoviscoelasticity. SIAM J. Math. Anal. 13 (1982), 397-408. MR653464 (83e:35104)

[9] C.M. Dafermos, L. Hsiao, Global smooth thermomechanical processes in one-dimensional nonlinear thermoviscoelasticity. Nonlinear Anal. 6 (1982), 435-454. MR661710 (83g:35059)

[10] C. Eck, Existence of solutions to a thermo-viscoelastic contact problem with Coulomb friction. Math. Models Methods Appl. Sci. 12 (2002), 1491-1511. MR1933934(2003h:74034)

[11] C. Eck, J. Jarušek, M. Krbec, Unilateral Contact Problems. Chapman \& Hall/CRC, Boca Raton, FL, 2005. MR2128865 (2006f:74049)

[12] A. Friedman, J. Nečas, Systems of nonlinear wave equations with nonlinear viscosity. Pacific J. Math. 135 (1988), 29-55. MR965683 (90b:35152)

[13] K.-H. Hoffmann, A. Zochowski, Existence of solutions to some non-linear thermoelastic systems with viscosity. Math. Methods in the Applied Sciences 15 (1992), 187-204. MR.1152708 (93d:73013)

[14] L. Hsiao, T. Luo, Large-time behavior of soutions to the equations of one-dimensional nonlinear thermoviscoelasticity. Quarterly Appl. Math. 56 (1998), 201-219. MR1622554(99h:73039)

[15] J. Jarušek, Solvability of a nonlinear thermo-viscoelastic contact problem with small friction and general growth of the heat energy. Preprint no. 273, Inst. für angew. Math., Uni. Erlangen, 2000.

[16] S. Jiang, Global large solutions to initial-boundary value problems in one-dimensional nonlinear thermoviscoelasticity. Quart. Appl. Math. 51 (1993), 731-744. MR1247437 (94h:35251)

[17] J.L. Lions, Quelques Méthodes de Résolution des Problèmes aux Limites non linéaires. Dunod, Paris, 1969. MR0259693(41:4326)

[18] J. Nečas, Dynamic in the nonlinear thermo-visco-elasticity. In: Symposium Partial Differential Equations Holzhau 1988 (Eds.: B.-W. Schulze, H. Triebel.), Teubner-Texte zur Mathematik 112, pp. 197-203. Teubner, Leipzig, 1989. MR1105810

[19] J. Nečas, A. Novotný, V. Šverák, Uniqueness of solution to the systems for thermoelastic bodies with strong viscosity. Math. Nachr. 149 (1990), 319-324. MR1124813 (92m:73025)

[20] M. Niezgódka, J. Sprekels, Existence of solutions for a mathematical model of structural phase transitions in shape memory alloys. Math. Methods in Appl. Sci. 10 (1988), 197-223 MR949654 (90d:73006)

[21] I. Pawłow, Three-dimensional model of thermomechanical evolution of shape memory material. Control and Cybernetics 29 (2000), 341-365. MR.1775170(2001f:74036)

[22] I. Pawłow, W.M. Zajączkowski, Global existence to a three-dimensional non-linear thermoelasticity system arising in shape memory materials. Math. Methods Appl. Sci. 28 (2005), 407-442. MR2114960 (2005h:35339) 
[23] I. Pawłow, A. Zochowski, Existence and uniqueness of solutions for a three-dimensional thermoelastic system. Dissertationes Math. (Rozprawy Mat.) 406 (2002), 46 pp. MR1926709(2004e:35221)

[24] R. Racke, S. Zheng: Global existence and assymptotic behavior in nonlinear thermoviscoelasticity. J. Diff. Eqns. 134 (1997), 46-67. MR1429091 (98a:35127)

[25] X. Ren, L. Truskinovsky, Finite scale microstructures in nonlocal elasticity. J. Elasticity 59 (2000), 319-355. MR 1833329 (2002c:74057)

[26] M.O. Rieger, J. Zimmer, Global existence for nonconvex thermoelasticity. Adv. Math. Sci. Appl. 15 (2005), 559-569. MR2198577 (2006i:74015)

[27] C. Rohde, Phase transitions and sharp-interface limits for the 1d-elasticity system with non-local energy. Interfaces and Free Boundaries 7 (2005), 107-129. MR2126146 (2005k:35268)

[28] T. Roubíček, Nonlinear Partial Differential Equations with Applications. Birkhäuser, Basel, 2005. MR2176645 (2007e:35002)

[29] W. Shen, S. Zheng, P. Zhu, Global existence and asymptotic behavior of weak solutions to nonlinear thermoviscoelastic systems with clamped boundary conditions. Quart. Appl. Math. 57 (1999), 93116. MR.1672183 (2000a:74063)

[30] J. Sprekels: Global existence for thermomechanical processes with nonconvex free energies of Ginzburg-Landau form. J. Math. Anal. Appl. 141, 333-348 (1989). MR.1009047 (90f:35191)

[31] J. Sprekels, S. Zheng, Global solutions to the equations of a Ginzburg-Landau theory for structural phase transitions in shape memory alloys. Physica D 39 (1989), 39-54. MR1021182 (91m:82097)

[32] S.J. Watson, A priori bounds in one-dimensional nonlinear thermoviscoelasticity. Contemporary Math. 255 (2000), 229-238. MR.1752511(2001d:35189)

[33] S.J. Watson, Unique global solvability for initial-boundary problems in one-dimensional nonlinear thermoviscoelasticity. Arch. Rational Mech. Anal. 153 (2000), 1-37. MR:1772535 (2001e:74038)

[34] J. Zimmer, Mathematische Modellierung und Analyse von Formgedächtnis legierungen in mehreren Raumdimensionen. Ph.D. Thesis, TU München, 2000.

[35] J. Zimmer, Global existence of a nonlinear system in thermoviscoelasticity with nonconvex energy. J. Math. Anal. Appl. 292 (2004), 589-604. MR2048273 (2004m:35261) 